\title{
ON CRITICAL POINT THEORY FOR INDEFINITE FUNCTIONALS IN THE PRESENCE OF SYMMETRIES
}

\author{
To the memory of Guido Stampacchia \\ BY \\ VIERI BENCI
}

\begin{abstract}
We consider functionals which are not bounded from above or from below even modulo compact perturbations, and which exhibit certain symmetries with respect to the action of a compact Lie group.

We develop a method which permits us to prove the existence of multiple critical points for such functionals. The proofs are carried out directly in an infinite dimensional Hilbert space, and they are based on minimax arguments.

The applications given here are to Hamiltonian systems of ordinary differential equations where the existence of multiple time-periodic solutions is established for several classes of Hamiltonians. Symmetry properties of these Hamiltonians such as time translation invariancy or evenness are exploited.
\end{abstract}

Introduction. Many variational problems which arise from physics or mathematical physics are indefinite in the sense that the functionals involved are not bounded from below or from above. However some of these functionals, defined in an appropriate function space, are "semidefinite" in the sense that they are bounded from below (or from above) if perturbed with a weakly continuous functional.

This paper deals with functionals which are not semidefinite. Usually problems involving indefinite functionals are more difficult to handle and only very recently a method has been developed which permits us to treat such problems directly in an infinite dimtnsional function space [7]. In [7] some theorems have been proved which establish the existence of at least one nontrivial critical point for such functionals.

In many physical situations there are problems which have symmetries with respect to the action of some Lie group. In this case we expect the existence of many critical points; this has been established for semidefinite functionals (cf. e.g. [8 and 3] for even functionals, [9] for a $\mathbf{Z}_{p}$-action with $p$ prime numbers [15, 16, 10 and 6] for an $S^{1}$-action).

In this paper we develop a method which allows us to estimate the number of critical points of indefinite functionals in the presence of symmetries. More precisely

Received by the editors July 16, 1980 and, in revised form, June 26, 1981.

1980 Mathematics Subject Classification. Primary 58E05, 70H05; Secondary 34C25.

Key words and phrases. Critical point, symmetry, minimax methods, Hamiltonian systems, periodic solutions. 
we consider a class of functionals defined on a real Hilbert space $H$ having the following form:

$$
f(u)=\frac{1}{2}\langle L u, u\rangle+\Phi(u)
$$

where $L$ is a bounded selfadjoint operator and $\Phi \in \mathcal{C}^{1}(H, \mathbf{R})$ is a functional whose Frechet derivative $\varphi=\Phi^{\prime} \in \bigodot^{0}(H, H)$ is a compact operator.

We assume also that both $L$ and $\varphi$ are equivariant with respect to the action of a linear representation of a Lie group. Before going on in our discussion we shall expose one of the theorems to be proved later on. It is not in the most general form, but it clarifies the nature of our results.

THEOREM 0.1. Let $c_{0}<c_{\infty}<0$ be two constants and let $f \in \mathcal{C}^{\mathrm{l}}(H, \mathbf{R})$ be a functional which satisfies the following assumptions:

$\left(\overline{\mathrm{f}}_{1}\right) f$ has the form (0-1).

$\left(\overline{\mathrm{f}}_{2}\right)$ Any sequence $\left\{u_{n}\right\} \subset H$ such that $f\left(u_{n}\right) \rightarrow c \in\left[c_{0}, c_{\infty}\right]$ and $\left\|f^{\prime}\left(u_{n}\right)\right\| \rightarrow 0$ as $n \rightarrow+\infty$ has a convergent subsequence.

$\left(\overline{\mathrm{f}}_{3}\right) \Phi(u)=\Phi(-u)$.

$\left(\overline{\mathrm{f}}_{4}\right)$ There are two closed subspaces of $H, \mathrm{H}^{+}$and $\mathrm{H}^{-}$, and a constant $\rho>0$ such that

(a) $f(u)>c_{0}$ for $u \in H^{+}$,

(b) $f(u)<c_{\infty}<0$ for $u \in H^{-} \cap S_{\rho}\left(S_{\rho}=\{u \in H \mid\|u\|=\rho\}\right)$.

Then the number of pairs of nontrivial critical points of $f$ is greater or equal to $\operatorname{dim}\left(H^{+} \cap H^{-}\right)-\operatorname{cod}\left(H^{-}+H^{+}\right)$. Moreover the corresponding critical values belong to $\left[c_{0}, c_{\infty}\right]$.

$\left(\overline{\mathrm{f}}_{2}\right)$ is a slightly weakened version of the well-known condition of Palais and Smale. $\left(\overline{\mathrm{f}}_{3}\right)$ expresses the equivariancy of the functional $f$ under the action of the antipodal map. (The action of more general Lie groups is considered in the paper.) $\left(\overline{\mathrm{f}}_{4}\right)$ is a geometrical condition which permits us to give a lower bound to the number of solutions.

The most interesting case occurs when both $\mathrm{H}^{+}$and $\mathrm{H}^{-}$are infinite dimensional; otherwise the functional $f$ would be semidefinite according to our definition and we would obtain a variation of known results. For example, if $\mathrm{H}^{+}$is finite dimensional, Theorem 0.1 is just a variant of Theorem 2.19 of Ambrosetti and Rabinowitz [3]. If $H^{-}$is finite dimensional, Theorem 0.1 is a variant of Theorem 12 of [8] (cf. also [6] in the case in which $f$ is invariant under an $S^{1}$-action).

Also note that Theorem 0.1 is similar to Theorem 0.1 of [7]. In [7] the form of the functional and the "geometry" are very similar, but no equivariancy property such as $\left(\bar{f}_{3}\right)$ is assumed. Therefore a weaker result is obtained, i.e. the existence of only one nontrivial solution. The proofs of our theorems are based on minimax arguments and they are carried out directly in an infinite dimensional Hilbert space.

This paper is organized as follows: In $\S 1$, we describe the abstract framework in which the theory will be constructed. This is done in order to clarify the main steps of the construction of the theory. For this purpose we introduce the concept of a "pseudoindex theory" which gives an axiomatic description of the properties required for the multiplicity results. 
When an "index theory" (in the sense of $[10,6,13,15]$ and Definition 1.1) is available, it is always possible to construct some pseudoindex theories. A pseudoindex theory is simply a tool which permits us to get rid of the indefiniteness. In this framework many of the known results for semidefinite functionals can be simplified and generalized (cf. e.g. [4]).

In $\$ 2$ we introduce two concrete "pseudoindex theories" which can both be applied to the study of indefinite functionals. In a sense which will become clear later on, they are duals of each other, and they give different characterizations of the critical values.

In order that the minimax principle be applicable, we need a suitable "deformation theorem" consistent with our pseudoindex theories. The proof of this theorem (Theorem 3.4) is the topic of §3. It is the central and most delicate part of this paper. Actually, the particular choice of our pseudoindex theories can be understood only in relation to the deformation theorem.

In $\S 4$ we can finally establish some abstract multiplicity results on the existence of critical points of (0-1) and in particular we shall prove Theorem 0.1.

In the last two sections we deal with applications.

In $\$ 5$ we look for periodic solutions of fixed period of asymptotically linear Hamiltonian systems. We suppose that the Hamiltonian function satisfies the following assumptions:

$$
\frac{\partial H}{\partial z}(z)=A z+o(|z|) \quad \text { as }|z| \rightarrow+\infty
$$

and

$$
\frac{\partial H}{\partial z}(z)=B z+o(|z|) \quad \text { as }|z| \rightarrow 0 .
$$

$z \in \mathbf{R}^{2 n}$ and $A, B: \mathbf{R}^{2 n} \rightarrow \mathbf{R}^{2 n}$ are linear symmetric operators.

We define a symplectic invariant $\vartheta(\tau B / 2 \pi, \tau A / 2 \pi)(\tau$ is the period we are interested in) which assumes only even values.

Under various assumptions on the Hamiltonian function, we show that the corresponding Hamiltonian system has at least $\frac{1}{2} \vartheta(\tau B / 2 \pi, \tau A / 2 \pi)$ nonconstant $\tau$-periodic solutions.

In the case in which $\vartheta(\tau B / 2 \pi, \tau A / 2 \pi) \geqslant 0$, our results are an improvement of those of Amann and Zehnder [1] since they required the Hamiltonian function to be strictly convex. If $\vartheta(\tau B / 2 \pi, \tau A / 2 \pi)<0$, we need a further symmetry property for the Hamiltonian function such as evenness. Then we get different results from theirs.

In the last sections we make some remarks on periodic solutions for superquadratic Hamiltonian functions and indicate how to apply the theory of $\S 4$ to such situations.

I thank P. H. Rabinowitz and E. Zehnder for helpful conversations.

1. The abstract framework. Let $X$ be a Riemannian manifold modelled on a Hilbert space $H$. For $A \subset X, \complement^{k}(A)$ denotes the space of $k$ times Fréchet differentiable maps from $A$ to $\mathbf{R}$. For $A$ and $B \subset X, C^{k}(A, B)$ denotes the sets of $k$ times Fréchet differentiable maps from $A$ to $B$. Id denotes the identity map. Moreover we set $N_{\delta}(A)=\{x \in X \mid \operatorname{dist}(x, A) \leqslant \delta\}$. 
Definition 1.1. An index theory $I$ on $X$ is a triplet $\{\Sigma, \Re, i\}$ which fulfills the following properties:

(1-1) $\Sigma$ is a family of closed subsets of $X$ such that $A \cup B, A \cap B, \overline{A \backslash B} \in \Sigma$ whenever $A, B \in \Sigma$.

(1-2) $\Re$ is a set of continuous mappings containing the identity and closed under composition.

(1-3) $\forall A \in \Sigma$, and $\forall h \in \mathscr{N}, \overline{h(A)} \in \Sigma$.

(1-4) $i: \Sigma \rightarrow \mathbf{N} \cup\{+\infty\}$ is a mapping which satisfies the following properties.

$(i-1) i(A)=0$ if and only if $A=\varnothing$.

(i-2) (Monotonicity) If $A \subset B$ then $i(A) \leqslant i(B) \forall A, B \in \Sigma$.

(i-3) (Subadditivity) $i(A \cup B) \leqslant i(A)+i(B) \forall A, B \in \Sigma$.

(i-4) (Continuity) If $A \in \Sigma$ is a compact set, then $\exists \delta>0$ such that $i\left(N_{\delta}(A)\right)=$ $i(A)$.

(i-5) (Supervariancy) $i(A) \leqslant i(\overline{h(A)}) \forall A \in \Sigma \forall h \in \mathfrak{T}$.

If $\tilde{\Sigma}$ is the family of all closed subsets of $X$, $\tilde{\mathscr{N}}$ is the family of the continuous mappings of $X$ homotopic to a constant map, and cat ${ }_{X}$ is the Ljiusternik-Schnirelmann category, then it is immediate to check that $\left\{\tilde{\Sigma}, \tilde{\mathscr{N}}\right.$, cat $\left._{X}\right\}$ defines an index theory on $X$.

In $\S 2$, we shall give other examples of index theories.

When we deal with indefinite functionals (i.e. functionals unbounded from below) the existence of an index theory may not be sufficient to guarantee the existence of critical points if we simply use the Ljiusternik-Schnirelmann theory in a direct way. Then the concept of pseudoindex theory turns out to be useful.

Definition 1.2. Let $X$ and $I=\{\Sigma, \mathfrak{R}, i\}$ be as in Definition 1.1. A pseudoindex theory $I^{*}$ is a couple $\left\{\mathscr{T}^{*}, i^{*}\right\}$ which satisfies the following assumptions

(1-6) $\Re * \subset \Re$ is a group of homeomorphisms of $X$ onto $X$.

(1-7) $i^{*}: \Sigma \rightarrow \mathbf{N} \cup\{+\infty\}$ is a map with the following properties

$\left(i^{*}-1\right) i^{*}(A) \leqslant i(A) \forall A \in \Sigma$.

$\left(i^{*}-2\right)$ If $A \subset B$, then $i^{*}(A) \leqslant i^{*}(B) \forall A, B \in \Sigma$.

$\left(i^{*}-3\right) i^{*}(\overline{A \backslash B}) \geqslant i^{*}(A)-i(B) \forall A, B \in \Sigma$.

$\left(i^{*}-4\right) i^{*}(h(A))=i^{*}(A) \forall h \in \mathfrak{N} * \forall A \in \Sigma$.

We shall show how the concept of pseudoindex can be applied in the search for critical points of a functional $f \in \mathcal{C}^{\mathrm{l}}(H)$.

For each $c \in \mathbf{R}$ we set

$$
\begin{aligned}
& \mathbb{Q}_{c}=\{x \in X \mid f(x) \leqslant c\}, \\
& \mathbf{K}_{c}=\left\{x \in X \mid f(x)=c \text { and } f^{\prime}(x)=0\right\} .
\end{aligned}
$$

Definition 1.3. If $f \in \mathrm{C}^{\mathrm{l}}(X)$ and $c_{0}, c_{\infty} \in \mathbf{R}$ (with $c_{0}<c_{\infty}$ ), we say that the triplet $\left\{f, c_{0}, c_{\infty}\right\}$ satisfies the property $(\mathrm{P})$ with respect to the couple $\left\{\Sigma, \Re^{*}\right\}$ if

$$
\begin{cases}\text { (a) } \mathbb{Q}_{c}, \mathbf{K}_{c} \in \Sigma \text { and } \mathbf{K}_{c} \text { is compact for every } c \in\left[c_{0}, c_{\infty}\right], \\ \text { (b) } \forall c \in\left[c_{0}, c_{\infty}\right], \forall N=N_{d}\left(\mathbf{K}_{c}\right), \exists \varepsilon>0 \text { and } \eta \in \Re^{*} \\ \\ \text { such that } \\ & \eta\left(\mathbb{Q}_{c+\varepsilon} \backslash N\right) \subset \mathbb{Q}_{c-\varepsilon} .\end{cases}
$$


In concrete cases, the property (P) is strictly related to the assumption (c) of Palais and Smale (cf. $\left(f_{2}\right)$, Theorem 3.4 and Corollary 3.5 ). The following theorem holds:

THEOREM 1.4. Let $X$ be a Riemannian manifold with an index theory $I=\{\Sigma$, গT, $i\}$ and a pseudoindex theory $I^{*}=\left\{\Re^{*}, i^{*}\right\}$. Suppose that $f \in \mathfrak{C}^{\mathrm{l}}(X)$ is a functional such that

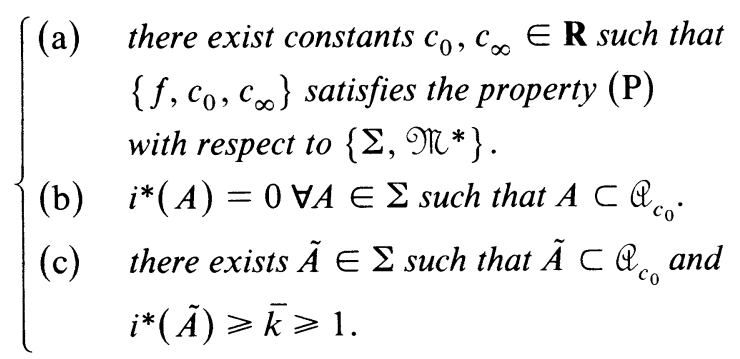

Then the real numbers

$$
c_{k}=\inf _{i^{*}(A) \geqslant k} \sup _{x \in A} f(x), \quad k=1, \ldots, \bar{k},
$$

are critical values of $f$ and $c_{0} \leqslant c_{1} \leqslant c_{2} \cdots \leqslant c_{k} \leqslant c_{\infty}$. Moreover if $c=c_{k}=\cdots=$ $c_{k+r}$ with $k \geqslant 1$ and $k+r \leqslant \bar{k}$ then $i\left(\mathbf{K}_{c}\right) \geqslant r+1$.

The proof of Theorem 1.4 follows standard arguments and it will not be given here.

REMARK 1.5. If in the assumption (c) we know that $i^{*}(\tilde{A})=+\infty$, then clearly (1-11) defines critical values for each $k \in \mathbf{N}^{+}$.

In order to apply Theorem 1.4 to concrete situations, it is necessary to construct an appropriate pseudoindex theory, which of course, depends on the functional $f$, whose critical points we seek.

In the following section we shall use a method to construct pseudoindex theories which is described by the following proposition.

Proposition 1.6. Let $I=\{\Sigma$, $\Re, i\}$ be an index theory on the Riemannian manifold $X$.

Let $\mathfrak{T} * \subset \mathfrak{T}$ be a group of homeomorphisms on $X$.

Given $Q \in \Sigma$, we set $i^{*}(A)=\min _{h \in \mathfrak{R}^{*}} i(h(A) \cap Q)$ for each $A \in \Sigma$.

Then $I^{*}=\left\{\mathfrak{T R}^{*}, i^{*}\right\}$ is a pseudoindex theory.

Proof. We have to show that the properties (1-7) are verified. $(i-1)(i-2)(i-4)$ are trivial. Let us prove ( $i-3)$. For each $h \in \Re^{*}$, and $A, B \in \Sigma$, we have

$$
h(\overline{A \backslash B}) \cap Q=(\overline{h(A) \backslash h(B)}) \cap Q=\overline{(h(A) \cap Q) \backslash h(B)}
$$

then applying $(i-3)$ and $(i-5)$, we get

$$
\begin{aligned}
i(h(\overline{A \backslash B}) \cap Q) & =i(\overline{(h(A) \cap Q) \backslash h(B)}) \\
& \geqslant i(h(A) \cap Q)-i(h(B))=i(h(A) \cap Q))-i(B)
\end{aligned}
$$


therefore

$$
\begin{aligned}
i^{*}(\overline{A \backslash B}) & =\min _{h \in \mathscr{N}^{*}} i(h(\overline{A \backslash B}) \cap Q) \\
& \geqslant \min _{h \in \mathscr{N}^{*}}[i(h(A) \cap Q)-i(B)]=i^{*}(A)-i(B) .
\end{aligned}
$$

In applications, when the appropriate index theory is already known, the main difficulty is to find an appropriate pseudoindex theory. Essentially the problem is to determine the right class of homomorphisms $\Re *$. This class should be "big" enough in order to contain a function $\eta$ such that $(1-9)(\mathrm{b})$ is satisfied. But if $\Re^{*}$ is "too big", it may happen that $i^{*}(A)=0$ or 1 for each $A \in \Sigma$ (cf. the construction of the pseudoindex theory of Proposition 1.6). Therefore Theorem 1.4 may not be applicable or gives the existence of only one critical value (cf. (1-10)(c)). In the next section we shall construct pseudoindex theories which will be useful in the search for critical values of functionals defined on a Hilbert space which are indefinite in the sense given in the introduction and which are symmetric with respect to the action of some Lie group.

However the abstract framework presented in this section can be applied to various situations and many known results about semidefinite functionals can be simplified and generalized. In [4] there is a detailed analysis of some of those situations.

2. Index and pseudoindex theories. From now on we shall consider only index and pseudoindex theories on a real Hilbert space $H$ on which the unitary representation $T_{g}$ of a compact Lie group $G$ acts. Some notation is now necessary.

$\langle\cdot, \cdot\rangle$ denotes the scalar product on $H$ and the symbol $($,$) will be left for the$ scalar product in $\mathbf{R}^{n}, B_{\rho}(u)$ denotes the closed ball of center $u$ and radius $\rho$. Also we set $B_{\rho}=B_{\rho}(0)$ and $S_{\rho}=\partial B_{\rho}$.

A functional $f \in \mathcal{C}^{\mathrm{l}}(H)$ is said to be $T_{g}$-invariant if

$$
f\left(T_{g} u\right)=f(u) \quad \forall u \in H, \forall g \in G .
$$

A map $h \in \mathcal{C}(H, H)$ is said to be $T_{g}$-equivariant if

$$
h\left(T_{g} u\right)=T_{g} h(u) \quad \forall u \in H, \forall g \in G .
$$

If $f \in \mathrm{C}^{\mathrm{l}}(H)$, then $f^{\prime} \in \mathrm{C}^{0}(H, H)$ since we identify $H$ with its dual, and if $f$ is $T_{g}$-invariant $f^{\prime}$ is $T_{g}$-equivariant. A subset $A \subset H$ is said to be $T_{g}$-invariant if

$$
T_{g} A=A \quad \forall g \in G .
$$

$F=\left\{u \in H \mid T_{g} u=u \forall g \in G\right\}$ will denote the linear space of the $T_{g}$-invariant points of $H$.

We recall (cf. e.g. [18]) that, by virtue of the Peter-Weyl theorem, a Hilbert space on which the representation of a compact Lie group acts may be decomposed in the following way:

$$
H=\bigoplus_{j=0}^{\infty} H_{j} \quad \text { where } H_{j} \text { is finite dimensional and invariant } \forall j \in \mathbf{N} .
$$

$V^{k}$ will denote a $k$-dimensional $T_{g}$-invariant subspace of $H$. 
Moreover we set

$$
\begin{aligned}
\Sigma\left(T_{g}\right) & =\{A \subset H \mid A \text { is closed and } T \text {-invariant }\}, \\
\mathfrak{M}\left(T_{g}\right) & =\{h \in \mathcal{C}(H, H) \mid h \text { is } T \text {-equivariant }\} .
\end{aligned}
$$

We shall say that an index theory $\{\Sigma, \mathscr{R}, i\}$ is related to the representation $T$ if $\Sigma=\Sigma\left(T_{g}\right)$ and $\mathscr{T}=\mathfrak{T}\left(T_{g}\right)$.

In this paper we shall consider only index theories related to some group representations.

In particular we shall consider only index theories which have an important property described by the following definition.

Definition 2.1. An index theory is said to satisfy the dimension property if there is a positive integer $d$ such that

$(2-2) i\left(V^{d k} \cap S_{1}\right)=k$ for all $d k$-dimensional subspaces $V^{d k} \in \Sigma$ such that $V^{d k} \cap$ $F=\{0\}$.

If an index theory has the dimension property, it is not difficult to prove that (cf. e.g. [4])

$\left(2-2^{\prime}\right) i(A) \leqslant k \quad$ if $A \subset V^{d k}$ and $A \cap F=\varnothing$ and

$\left(2-2^{\prime \prime}\right) i(K)<+\infty$ if $K$ is compact and $K \cap F=\varnothing$.

It is not difficult to realize that any index theory with the dimension property has the further property that

(2-3) $i(A)=+\infty \quad$ whenever $A \cap F \neq \varnothing$.

In fact, if $\bar{u} \in F$, the constant map $\psi_{1}: H \rightarrow\{\bar{u}\}$ belongs to $\Re$. Then, if $\bar{u} \in A$, by $(i-5)$ and (2-2), we have that

$$
i(\{\bar{u}\}) \geqslant i\left(\psi_{1}\left(H \cap S_{1}\right)\right) \geqslant i\left(H \cap S_{1}\right) \geqslant d^{-1} \operatorname{dim} H .
$$

Since, in general, $\operatorname{dim} H=+\infty,(2-3)$ follows.

The property (2-3) causes some problems when multiplicity results need to be proved. This difficulty sometimes may be overcome by constructing appropriate pseudoindex theories and using some further tricks. All these remarks will become clear in the next sections.

We shall give three examples of index theories related to the representations of some Lie group which satisfy the dimension property with $d=1,2$ and 4 respectively. The first two examples will be used in the applications in the last sections.

I ExAmple. Consider the group $\mathbf{Z}_{2}=\{0,1\}$ and the unitary representation of this group on a real Hilbert space $H$ defined as follows

$$
T_{0} u=u, \quad T_{1} u=-u, \quad u \in H .
$$

If $A \in \Sigma\left(T_{g}\right)=$ \{closed subsets of $H$ symmetric with respect to the origin $\}$ we set

$$
\gamma(A)=k
$$

if $k$ is the smallest integer such that there exists a continuous odd map

$$
\Phi: A \rightarrow \mathbf{R}^{k} \backslash\{0\} .
$$

If such a map does not exist we set $i(A)=+\infty$ and we set $\gamma(\varnothing)=0$. This set function called "genus" has been introduced by Krasnoselskii in an equivalent form 
(see [11]) and used by many authors (cf. e.g. [3, 4, 8, 13]). In [11] and in [13] it has been proved that $\left\{\Sigma\left(T_{g}\right), \Re\left(T_{g}\right), \gamma\right\}$ is an index theory and that it satisfies the dimension property with $d=1$.

Actually in the papers mentioned above the genus has been defined only for those sets $A \in \Sigma$ such that $0 \notin A$, but it can be extended to all $\Sigma$. In fact if $0 \in A,(2-3)$ shows $i(A)=+\infty$ and that all the required properties are satisfied.

II EXAMPLE. Consider the group $S^{1}=\{z \in \mathbf{C}|| z \mid=1\}$ and a unitary representation $T_{z}$ of this group on a real Hilbert space $H$. To simplify the notation we shall write $T_{s}$ instead of $T_{z}$ if $z=e^{i s}(s \in[0,2 \pi))$. If $A \in \Sigma\left(T_{s}\right)=$ closed $T_{s}$-invariant subsets of $H\}$ we set $\tau(A)=k$ if $k$ is the smallest integer for which there exist a number $n \in \mathbf{N}^{+}$and a continuous map

$$
\Phi: A \rightarrow \mathbf{C}^{k} \backslash\{0\}
$$

such that

$$
\Phi\left(T_{s} u\right)=e^{i n s} \Phi(u) \quad \forall u \in A, \forall s \in[0,2 \pi]
$$

If such a map does not exist we set $\tau(A)=+\infty$; moreover we set $\tau(\varnothing)=0$. In [6] it has been proved that $\left\{\Sigma\left(T_{s}\right), \mathfrak{N}\left(T_{s}\right), i\right\}$ is an index theory.

If $V^{k^{\prime}} \in \Sigma$ is an invariant subspace of $H$, and $V^{k^{\prime}} \cap F=\{0\}$ it is not difficult to see that its dimension is even i.e. $k^{\prime}=2 k, k \in \mathbf{N}$. In [6] it has been proved that the above index theory satisfies the dimension property with $d=2$. Since all the invariant spaces are even dimensional this makes sense.

III EXAMPLE. Let $H$ be the set of all sequences of quaternions $\left\{a_{j}\right\}_{j \in \mathbf{N}}\left(a_{j} \in \mathbf{H}\right)$ such that

$$
\sum_{j=1}^{\infty}\left|a_{j}\right|^{2}<+\infty
$$

Clearly $H$ has the natural structure of Hilbert space on the real field if we identify $\mathbf{H}$ with $\mathbf{R}^{4}$.

A unitary representation of

$$
S^{3}=\{w \in \mathbf{H} \| w \mid=1\}
$$

defined in the natural way acts on $H$ :

$$
T_{w}\left(\left\{a_{j}\right\}_{j \in \mathbf{N}}\right)=\left\{w a_{j}\right\}_{j \in \mathbf{N}}
$$

In [10] it has been proved that there exists an index theory related to the above representation with $d=4$. The index theories $\gamma$ and $\tau$ will be used in the applications of $\S \S 5$ and 6 . In the literature there are many other ones, perhaps with different names. We mention only the index of Fadell and Rabinowitz [10] and we refer to their paper for further information on this topic.

Our program now is to construct two pseudoindex theories $I_{1}^{*}$ and $I_{2}^{*}$ related to any index theory which satisfies the dimension property. As has already been 
observed these pseudoindex theories will permit us to deal with indefinite functionals. To begin this program, we define the following families of maps. For $X$ is a Banach space, we set

$$
\begin{aligned}
& \Gamma_{0}(X)=\{h \in \mathcal{C}(X, X) \mid h=U+\varphi \text { where } U \text { is linear and } \varphi \text { is compact }\}, \\
& \Gamma_{1}(X)=\left\{h=U+\varphi \in \Gamma_{0} \mid h \text { and } U \text { are homeomorphisms }\right\} .
\end{aligned}
$$

Lemma 2.2. Let $h=U+\varphi \in \Gamma_{1}(X)$. Then

$$
\psi=h^{-1}-U^{-1}
$$

is compact.

Proof. Set $v=h(u)$, so that

$$
\begin{aligned}
u & =h^{-1}(v)=U^{-1}(v)+\psi(v)=U^{-1} \circ h(u)+\psi(v) \\
& =U^{-1}(U u+\varphi(u))+\psi(v)=u+U^{-1} \circ \varphi(u)+\psi(v)
\end{aligned}
$$

Then

$$
\psi(v)=-U^{-1} \circ \varphi(u)=-U^{-1} \circ \varphi \circ h^{-1}(v)
$$

and since $\varphi$ is compact, it follows that $\psi$ is compact.

The pseudoindex theories we are going to construct are related to a linear $T_{g}$-invariant subspace $H^{+} \subset H, \quad H^{+} \in \Sigma$. The most interesting case occurs when both the dimension and the codimension of $\mathrm{H}^{+}$are infinite. Otherwise, our pseudoindex theories would give results for semidefinite functionals, but these functionals could be treated in a simpler and more general way (cf. [4, 13]).

Let $\mathcal{Q}$ be a group of linear homeomorphisms such that

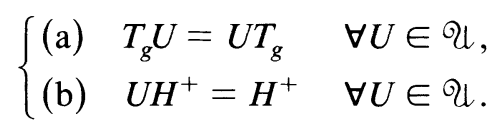

In our applications to the functional (0-1), we shall suppose that $H^{+}$is $L$-invariant and we shall set

$$
\text { थ }=\left\{e^{t L} \mid t \in \mathbf{R}\right\}
$$

However, in the construction of our pseudoindex theory, every family $\mathscr{Q}$ which satisfies (2-4) works as well.

Let $\Re^{*}$ denote a class of mappings $h$ such that

$$
\begin{aligned}
& \text { (a) } h \in \Re \text { i.e. } h \text { is } T_{g} \text {-equivariant, } \\
& \text { (b) } h \in \Gamma_{1}(H) \text { i.e. } h \text { is a homeomorphism of the } \\
& \text { form } U+\varphi \text { where } \varphi \text { is compact, and } \\
& \text { (c) } U \in \mathcal{Q} \text {. }
\end{aligned}
$$

By Lemma 2.2, the following corollary is straightforward.

COROLlaRY 2.3. $\Re^{*}$ is a group of homeomorphisms.

If we suppose that $\mathrm{F} \subset \mathrm{H}^{+}$we set

$$
I_{1}^{*}=\left\{\Re^{*}, i_{1}^{*}\right\} \quad \text { where } i_{1}^{*}(A)=\min _{h \in \mathscr{N}^{*}} i\left(h(A) \cap H^{+}\right) .
$$


Then by virtue of Corollary 2.3 and proof $1.6, I_{1}^{*}$ is a pseudoindex theory. In order that this pseudoindex theory be useful it is necessary to show that it is not trivial, i.e. that there are sets of any pseudoindex.

We shall prove the following theorem.

THEOREM 2.4. Let $H^{-}, H^{+} \in \Sigma$ be linear subspaces of $H$ such that $\operatorname{cod}\left(H^{-}+H^{+}\right)$ $<+\infty, \operatorname{dim}\left(H^{+} \cap H^{-}\right)<+\infty$ and $F \subset H^{+}$. Then

$$
i_{1}^{*}\left(H^{-} \cap S_{\rho}\right)=\frac{1}{d}\left[\operatorname{dim}\left(H^{+} \cap H^{-}\right)-\operatorname{cod}\left(H^{+}+H^{-}\right)\right] .
$$

Before proving Theorem 2.4, we shall introduce another pseudoindex theory which will be useful in different situations.

If we suppose that $H^{+} \cap F=\{0\}$, we fix a constant $\rho>0$, and we set

$$
I_{2}^{*}=\left\{\mathscr{R}^{*}, i_{2}^{*}\right\} \quad \text { where } i_{2}^{*}=\min _{h \in \mathscr{R}^{*}} i\left(h(A) \cap S_{\rho} \cap H^{+}\right) .
$$

Also in this case, Corollary 2.3 and Proposition 1.6 show that (2-8) defines a pseudoindex theory. The following nontriviality theorem holds.

THEOREM 2.5. Let $H^{-}, H^{+} \in \Sigma$ be a linear subspace of $H$. Suppose that $F \cap H^{+}=$ $\{0\}, F \subset H^{-}, \operatorname{dim}\left(H^{+} \cap H^{-}\right)<+\infty ; \operatorname{cod}\left(H^{+}+H^{-}\right)<+\infty$ and that $H^{-}$is invariant for every $U \in \mathcal{Q}$. Then

$$
i_{2}^{*}\left(H^{-}\right)=\frac{1}{d}\left[\operatorname{dim}\left(H^{+} \cap H^{-}\right)-\operatorname{cod}\left(H^{+}+H^{-}\right)\right] .
$$

In order to prove Theorem 2.4 and Theorem 2.5 some work is necessary.

LEMMA 2.6. Let $V, W \in \Sigma$ be two invariant subspaces of $H$ such that

$$
F \subset V \subset W \text { and } \operatorname{cod}_{W} V<+\infty \text {. }
$$

If $A \in \Sigma$ is a bounded subset of $W$, then

$$
i(A \cap V) \geqslant i(A)-\frac{1}{d} \operatorname{cod}_{W} V .
$$

Proof. In order to simplify the notation we set $k=i(A) ; k_{1}=d^{-1} \operatorname{dim} V_{1}$ where $V_{1}$ is the orthogonal complement of $V$ in $W$.

Now we argue indirectly and we suppose that

$$
i(A \cap V) \leqslant k-k_{1}-1 \text {. }
$$

By (i-4) there exists a neighborhood $N=N_{\delta}(A \cap V)$ such that

$$
i(N) \leqslant k-k_{1}-1 \text {. }
$$

We set

$$
A_{1}=A \cap N, \quad A_{2}=\overline{A \backslash N}
$$

Then we have

$$
\operatorname{dist}\left(A_{2}, V\right) \geqslant \delta>0 .
$$

If $P$ denotes the orthogonal projector on $V_{1}$, by $(i-5),\left(2.2^{\prime}\right)$, and the above inequality,

$$
i\left(A_{2}\right) \leqslant i\left(P A_{2}\right) \leqslant \frac{1}{d} \operatorname{dim} V_{1}=k_{1} .
$$


Then using (i-3), (i-2) and the above inequalities, we get

$$
i(A) \leqslant i\left(A_{1}\right)+i\left(A_{2}\right) \leqslant i(N)+i\left(A_{2}\right) \leqslant\left(k-k_{1}-1\right)+k_{1}=k-1
$$

and this contradicts our assumptions. Thus the lemma is proved.

LEMmA 2.7. Suppose that $H=\bigoplus_{i=1}^{4} H_{i}$ where $H_{i}(i=1, \ldots, 4)$ are four mutually orthogonal subspaces of $H$. Moreover suppose that $\operatorname{dim} H_{i}<+\infty$ for $i=2,4$ and that $\mathrm{F} \subset \mathrm{H}_{3}$. Let

$$
\mathscr{F}=\{\Phi \in \mathscr{T} \mid \Phi=\mathrm{Id}+\mathrm{g} \text { where } \mathrm{g} \text { has finite dimensional range }\}
$$

then

$$
i\left(\Phi\left(A_{\rho}\right) \cap\left(H_{2} \oplus H_{3}\right)\right) \geqslant \frac{1}{d}\left[\operatorname{dim} H_{2}-\operatorname{dim} H_{4}\right]
$$

where

$$
A_{\rho}=S_{\rho} \cap\left(H_{1} \oplus H_{2}\right)
$$

Proof. Given $\Phi=\mathrm{Id}+g$, let $W_{0}$ be a finite dimensional space such that $W_{0} \supset g\left(A_{\rho}\right)$ and we set

$$
V_{1}=W_{0} \cap H_{1}
$$

and

$$
W=V_{1} \oplus H_{2} \oplus H_{3} \oplus H_{4}
$$

Then we have

$$
\begin{aligned}
\Phi\left(A_{\rho}\right) \cap\left(H_{2} \oplus H_{3}\right) & \supseteq \Phi\left(A_{\rho} \cap W\right) \cap\left(H_{2} \oplus H_{3}\right) \\
& \supseteq\left[\Phi\left(A_{\rho} \cap W\right) \cap W\right] \cap\left(H_{2} \oplus H_{3}\right) .
\end{aligned}
$$

By the definition of $W$

$$
\Phi\left(A_{\rho} \cap W\right) \subset W
$$

so by the above formula, (i-5) and (2-2)

$$
\begin{aligned}
i\left(\Phi\left(A_{\rho} \cap W\right) \cap W\right) & =i\left(\Phi\left(A_{\rho} \cap W\right)\right) \geqslant i\left(A_{\rho} \cap W\right) \\
& =i\left(S_{\rho} \cap\left(V_{1} \oplus H_{2}\right)\right)=\frac{1}{d} \operatorname{dim} V_{1}+\frac{1}{d} \operatorname{dim} H_{2} .
\end{aligned}
$$

On the other hand we have that

$$
\operatorname{cod}_{W}\left(H_{2} \oplus H_{3}\right)=\operatorname{dim} V_{1}+\operatorname{dim} H_{4} .
$$

Using Lemma 2.6, (2-10), (2-11) and (2-12) we get

$$
\begin{aligned}
i\left(\Phi\left(A_{\rho}\right) \cap\left(H_{2} \oplus H_{3}\right)\right) & \geqslant i\left(\Phi\left(A_{\rho} \cap W\right) \cap W\right)-\frac{1}{d} \operatorname{cod}_{W}\left(H_{2} \oplus H_{3}\right) \\
& \geqslant\left(\frac{1}{d} \operatorname{dim} V_{1}+\frac{1}{d} \operatorname{dim} H_{2}\right)-\left(\frac{1}{d} \operatorname{dim} V_{1}+\frac{1}{d} \operatorname{dim} H_{4}\right) \\
& =\frac{1}{d}\left(\operatorname{dim} H_{2}-\operatorname{dim} H_{4}\right) .
\end{aligned}
$$


Lemma 2.8. Let $H, H_{i}(i=1,2,3,4)$ and $A_{\rho}$ be as in Lemma 2.7 and set $\mathscr{K}=\{\Phi \in \mathfrak{K} \mid \Phi=\mathrm{Id}+\varphi$ where $\varphi$ is compact and $\Phi$ is a homeomorphism $\}$ then

$$
i\left(\Phi\left(A_{\rho}\right) \cap\left(H_{2} \oplus H_{3}\right)\right) \geqslant \frac{1}{d}\left[\operatorname{dim} H_{2}-\operatorname{dim} H_{4}\right] .
$$

Proof. First of all we shall prove that the set

$$
K=\Phi\left(A_{\rho}\right) \cap\left(H_{2} \oplus H_{3}\right)=\left\{\left[S_{\rho} \cap\left(H_{1} \oplus H_{2}\right)\right]+\Phi\left(A_{\rho}\right)\right\} \cap\left(H_{2} \oplus H_{3}\right)
$$

is compact. Consider the sequence $u_{n} \in K$. Then we can write

$$
u_{n}=v_{n}+w_{n}+z_{n}
$$

with $v_{n} \in H_{1}, w_{n} \in H_{2}, z_{n}=\varphi\left(w_{n}+v_{n}\right)$ and $v_{n}+w_{n} \in S_{\rho}$. Since $H_{2}$ is finite dimensional and $w_{n}$ is bounded then we can suppose that $w_{n}$ converges (possibly considering a subsequence). Since $\varphi$ is a compact map we can suppose that $z_{n}$ converges. Let $P_{i}$ be the orthogonal projector on $H_{i}$. Applying $P_{1}$ to (2-14) we get $0=v_{n}+P_{1} z_{n}$. Thus, since $P_{1} z_{n}$ converges, we deduce that $v_{n}$ converges too. Therefore $u_{n}$ converges and $K$ is compact.

By virtue of $(i-4)$ there is a constant $\delta_{1}>0$ such that

$$
i\left(\Re_{\delta_{1}}\right)=i\left(\Phi\left(A_{\rho}\right) \cap\left(H_{2} \oplus H_{3}\right)\right)
$$

where

$$
\Re_{\alpha}=N_{\alpha}\left(\Phi\left(A_{\rho}\right) \cap\left(H_{2} \oplus H_{3}\right)\right) .
$$

We set $B=\Phi^{-1}\left(\Re_{\delta_{1 / 4}}\right)$. Clearly we have that

$$
\Phi\left(\overline{A_{\rho} \backslash B}\right) \cap\left(H_{2} \oplus H_{3}\right)=\varnothing .
$$

We claim that there exists a constant $\delta_{2}>0$ such that

$$
\left\|P_{1,4} u\right\| \geqslant \delta_{2} \forall u \in \Phi\left(\overline{A_{\rho} \backslash B}\right) \text { where } P_{1,4}=P_{1}+P_{4} .
$$

In order to prove (2-17) we argue indirectly and we suppose that there exists a sequence $\left\{u_{n}\right\} \subset \Phi\left(\overline{A_{\rho} \backslash B}\right)$ such that $P_{1,4} u_{n} \rightarrow 0$. As in (2-14) we set $u_{n}=v_{n}+w_{n}+$ $z_{n}$ with $v_{n} \in H_{1}, w_{n} \in H_{2}$ and $z_{n}=\varphi\left(u_{n}+w_{n}\right)$. Then we have

$$
P_{1,4} u_{n}=v_{n}+P_{1,4} z_{n} \text {. }
$$

Since $P_{1,4} u_{n}$ and $P_{1,4} z_{n}$ are convergent sequences (possibly considering subsequences) then also $v_{n}$ converges, and since $w_{n}$ converges, $u_{n}$ converges to a point $\bar{u} \in \Phi\left(\overline{A_{\rho} \backslash B}\right)$ (we recall that $\Phi\left(\overline{A_{\rho} \backslash B}\right)$ is a closed set since $\Phi$ is a homeomorphism). Since $P_{1,4} \bar{u}=\lim _{n \rightarrow \infty} P_{1,4} u_{n}=0$, then $\bar{u} \in H_{2} \oplus H_{3}$. Therefore

$$
\bar{u} \in \Phi\left(\overline{A_{\rho} \backslash B}\right) \cap\left(H_{2} \oplus H_{3}\right)
$$

and this fact contradicts (2-16). Then (2-17) is proved. Now let $g$ be a finite dimensional equivariant map such that

$$
\|g(u)-\varphi(u)\| \leqslant \delta \quad \forall u \in A_{\rho} \text { where } \delta=\frac{1}{2} \min \left(\delta_{1}, \delta_{2}\right) \text {. }
$$


Such a map can be easily constructed by virtue of (2-1). If we set $\tilde{\Phi}=\mathrm{Id}+g$, then $\tilde{\Phi} \in \mathscr{F}(\mathscr{F}$ is defined in Lemma 2.7) and

$$
\|\Phi(u)-\tilde{\Phi}(u)\| \leqslant \delta \quad \forall u \in A_{\rho} .
$$

It is easy to see that

$$
\tilde{\Phi}\left(\overline{A_{\rho} \backslash B}\right) \cap\left(H_{2} \oplus H_{3}\right)=\varnothing .
$$

In fact, if $z \in \overline{A_{\rho} \backslash B}$, by (2-17) and (2-18), we get

$$
\begin{aligned}
\left\|P_{1,4} \tilde{\Phi}(z)\right\| & \geqslant\left\|P_{1,4} \Phi(z)\right\|-\left\|P_{1,4}(\Phi(z)-\tilde{\Phi}(z))\right\| \\
& \geqslant \delta_{2}-\|\Phi(z)-\tilde{\Phi}(z)\| \geqslant \frac{\delta_{2}}{2}>0 .
\end{aligned}
$$

Using (2-19), we have that

$$
\tilde{\Phi}\left(A_{\rho}\right) \cap\left(H_{2} \oplus H_{3}\right)=\tilde{\Phi}\left(A_{\rho} \cap B\right) \cap\left(H_{2} \oplus H_{3}\right) .
$$

Also by (2-18) we have that

$$
\tilde{\Phi}(B) \subset \Re_{\delta_{1}} .
$$

By the above formula and (2-20), we get

$$
\tilde{\Phi}\left(A_{\rho}\right) \cap\left(H_{2} \oplus H_{3}\right) \subset \mathfrak{K}_{\delta_{1}} .
$$

Therefore using (2-15), (i-2) and Lemma 2.7 we have that

$$
\begin{gathered}
i\left(\Phi\left(A_{\rho}\right) \cap\left(H_{2} \oplus H_{3}\right)\right)=i\left(\Re_{\delta_{1}}\right) \geqslant i\left(\tilde{\Phi}\left(A_{\rho}\right) \cap\left(H_{2} \oplus H_{3}\right)\right) \\
\geqslant d^{-1}\left(\operatorname{dim} H_{2}-\operatorname{dim} H_{4}\right) .
\end{gathered}
$$

Proof of Theorem 2.4. If $H^{-} \cap F \neq\{0\}$ then, since $F$ is $h$-invariant

$$
h\left(H^{-} \cap S_{\rho}\right) \cap F \neq \varnothing \forall h \in \Re .
$$

Therefore, by $(i-2), i\left(h\left(H^{-} \cap S_{\rho}\right) \cap H^{+}\right) \geqslant i\left(h\left(H^{-} \cap S_{\rho}\right) \cap F\right)=+\infty$ and Theorem 2.4 is proved. If $H^{-} \cap F=\{0\}$, then we set

$H_{2}=H^{+} \cap H^{-}$,

$H_{1}=$ orthogonal complement of $H_{2}$ in $H^{-}$,

$\mathrm{H}_{3}=$ orthogonal complement of $\mathrm{H}_{2}$ in $\mathrm{H}^{+}$,

$H_{4}=$ orthogonal complement of $H_{1} \oplus H_{2} \oplus H_{3}$ in $H$.

Because of our assumptions $F \subset H_{3}$. Then the assumptions of Lemma 2.8 are satisfied. For every $h=U+\varphi \in \mathfrak{T}^{*}$, we have

$$
\begin{aligned}
i\left(h\left(H^{-} \cap S_{\rho}\right) \cap H^{+}\right) & =i\left(U^{-1} \circ h\left(H^{-} \cap S_{\rho}\right) \cap U^{-1} H^{+}\right) \quad \text { by }(i-5) \\
& =i\left(\left(\mathrm{Id}+U^{-1} \circ \varphi\right)\left(H^{-} \cap S_{\rho}\right) \cap H^{+}\right) \quad \text { by }(2-4)(\mathrm{b}) \\
& =i\left(\left(\mathrm{Id}+U^{-1} \circ \varphi\right)\left(\left(H_{1} \oplus H_{2}\right) \cap S_{\rho}\right) \cap\left(H_{2} \oplus H_{3}\right)\right) .
\end{aligned}
$$

Since Id $+U^{-1} \circ \varphi \in \mathscr{K}$, by Lemma 2.8, we have that

$$
i\left(h\left(H^{-} \cap S_{\rho}\right) \cap H^{+}\right) \geqslant \frac{1}{d}\left[\operatorname{dim} H_{2}-\operatorname{dim} H_{4}\right] \quad \forall h \in \Re^{*} .
$$


Since $\operatorname{dim} H_{2}=\operatorname{dim} H^{+} \cap H^{-}$and $\operatorname{dim} H_{4}=\operatorname{cod} H^{+}+H^{-}$, by the definition of $i_{1}^{*}$ the conclusion follows.

ProOF OF THEOREM 2.5. We set

$H_{2}=H^{+} \cap H^{-}$,

$H_{1}=$ orthogonal complement of $H_{2}$ in $H^{+}$,

$\mathrm{H}_{3}=$ orthogonal complement of $\mathrm{H}_{2}$ in $\mathrm{H}^{-}$,

$H_{4}=$ orthogonal complement of $H_{1} \oplus H_{2} \oplus H_{3}$ in $H$.

Since $F \cap H^{+}=\{0\}$ and $F \subset H^{-}$, then $F \subset H_{3}$. Then the assumptions of Lemma 2.8 are satisfied.

For every $h=U+\varphi \in \Re^{*}$, we have

$$
i\left(h\left(H^{-}\right) \cap S_{\rho} \cap H^{+}\right)=i\left(H^{-} \cap h^{-1}\left(S_{\rho} \cap H^{+}\right)\right) \text {by }(i-5) .
$$

By Lemma $2.2, h^{-1}=U^{-1}+\psi$ with $\psi$ compact. Since $U H^{-}=H^{-}$we have

$$
\begin{aligned}
i\left(h\left(H^{-}\right) \cap S_{\rho} \cap H^{+}\right) & =i\left(H^{-} \cap\left(U^{-1}+\psi\right)\left(S_{\rho} \cap H^{+}\right)\right) \\
& =i\left(U H^{-} \cap(\operatorname{Id}+U \circ \psi)\left(S_{\rho} \cap H^{-}\right)\right) \quad \text { by }(i-5) \\
& =i\left(H_{2} \oplus H_{3} \cap(\operatorname{Id}+U \circ \psi)\left(S_{\rho} \cap H_{1} \oplus H_{2}\right)\right) .
\end{aligned}
$$

Since Id $+U \circ \varphi \in \mathscr{K}$, by Lemma 2.8, we have that

$$
\begin{aligned}
i\left(h\left(H^{-}\right) \cap S_{\rho} \cap H^{+}\right) & \geqslant \frac{1}{d}\left[\operatorname{dim} H_{2}-\operatorname{dim} H_{4}\right] \\
& =\frac{1}{d}\left[\operatorname{dim}\left(H^{+} \cap H^{-}\right)-\operatorname{cod}\left(H^{+}+H^{-}\right)\right] \quad \forall h \in \Re * .
\end{aligned}
$$

Since for $h=$ Id, we get the equality, the conclusion follows from the definition of $i_{2}^{*}$.

3. A deformation theorem. In $\S 2$ we have constructed two pseudoindex theories. If we want to apply Theorem 1.4, we need to characterize the functions $f \in \mathcal{C}^{1}(H)$ which satisfy the property $(\mathrm{P})$ with respect to such pseudoindex theories. This is the aim of this section. Clearly the main difficulty is to find a function $\eta$ as in (1-9)(b). As usual this function will be a deformation, i.e. $\eta(u)=\mathscr{U}\left(t_{0}, u\right)$ where $\mathscr{U}$ is a flow on $H$ and $t_{0}$ a fixed real number. This flow will be related to a vector field which is a suitable approximation of the vector field $-f^{\prime}$. In our case, the main difficulty in carrying out this program is the fact that $\eta$ must belong to $\mathfrak{R}^{*}$; therefore the construction of the flow $U(t, u)$ will involve some technicalities which will be treated in this section. We shall start by proving some lemmas to be used later in the construction of $\mathcal{Q}$.

Lemma 3.1. Let $X$ and $Y$ be two Banach spaces and let $f: X \rightarrow Y$ be a locally Lipschitz continuous function. Then any compact set $K \subset X$ has a neighborhood $\Re$ such that $\left.f\right|_{g}$ is (uniform) Lipschitz continuous.

PROOF. For each $u \in K$, there exist an open ball $B(u, \delta(u))$ and a constant $l(u)$ such that

$$
\|f(v)-f(w)\| \leqslant l(u)\|v-w\|, \quad \forall v, w \in B(u, \delta(u)) .
$$


The family of sets $\left\{B\left(u, \frac{1}{2} \delta(u)\right)\right\}_{u \in \mathbf{K}}$ is an open covering of $K$. Therefore we can extract a finite covering

$$
\left\{B\left(u_{i}, \frac{1}{2} \delta\left(u_{i}\right)\right)\right\}_{i \in I} \text { where } I \text { is a finite set of indices. }
$$

We now set

$$
\Re=\bigcup_{i \in I} B\left(u_{i}, \frac{1}{2} \delta\left(u_{i}\right)\right) \text { and } M=\sup _{u \in f(\Re)}\|u\| .
$$

$M$ is less than infinity by the compactness of $K$ and the construction of $\mathscr{N}$. We set

$$
l=\max \left\{\frac{4 M}{\delta}, \max _{i \in I} l\left(u_{i}\right)\right\} \quad \text { where } \delta=\min _{i \in I} \delta\left(u_{i}\right) .
$$

We claim that

$$
\|f(v)-f(w)\| \leqslant l\|v-w\| \text { for each } v, w \in \Re .
$$

In order to prove the above inequality we distinguish two cases

First case. $\|v-w\| \geqslant \delta / 2$. Then we have

$$
\|f(v)-f(w)\| \leqslant\|f(v)\|+\|f(w)\| \leqslant 2 M \leqslant \frac{4 M}{\delta} \cdot \frac{\delta}{2} \leqslant l\|v-w\| .
$$

Second case. $\|v-w\|<\delta / 2$. Suppose that $v \in B\left(u_{i}, \frac{1}{2} \delta\left(u_{i}\right)\right)$. Then $w$ and $v \in$ $B\left(u_{i}, \delta\left(u_{i}\right)\right)$. Therefore we have

$$
\|f(v)-f(w)\| \leqslant l\left(u_{i}\right)\|v-w\| \leqslant l\|v-w\| .
$$

LeMMA 3.2. Let $\varphi: H \rightarrow H$ be a compact operator. Then, given $\gamma>0$, there exists an operator $\tilde{\varphi}: H \rightarrow H$ which satisfies the following assumptions:

$$
\left\{\begin{array}{l}
\text { (a) } \tilde{\varphi} \text { is compact, } \\
\text { (b) } \tilde{\varphi} \text { is locally Lipschitz continuous, } \\
\text { (c) }\|\varphi(u)-\tilde{\varphi}(u)\| \leqslant \gamma .
\end{array}\right.
$$

Moreover, if $\varphi$ is $T_{g}$-equivariant, $\tilde{\varphi}$ can be chosen to be equivariant.

Proof. For each $w \in H$, we set $\mathscr{X}_{w}=\{u \in H \mid\|\varphi(u)-\varphi(w)\|<\gamma$ and $\|u-w\|$ $<1\} .\left\{\mathscr{T}_{w}\right\}_{w \in H}$ is an open covering of $H$. Therefore there exist a locally finite refinement $\mathscr{T}_{i}$ and points $w_{i}$ such that $\mathscr{T}_{i} \subset \mathscr{N}_{w_{i}}$. Let $\rho_{i}(u)$ denote the distance of $u$ to the complement of $\mathfrak{l}_{i}$. Then $\rho_{i}$ is Lipschitz continuous and vanishes outside of $\Re_{i}$. Let

$$
\beta_{i}(u)=\rho_{i}(u) / \sum_{j \in I} \rho_{j}(u)
$$

Since $\left\{\mathcal{T}_{i}\right\}_{i \in I}$ is a locally finite covering, for each $u \in H$ the denominator of $\beta_{i}(u)$ is a finite sum and the above formula makes sense. Moreover the $\beta_{i}$ 's are Lipschitz continuous and

$$
\sum_{i} \beta_{i}(u)=1
$$

By the definition of the $\mathscr{N}_{\boldsymbol{w}_{i}}$ 's and the $\mathfrak{X}_{i}$ 's, we have that

$$
\left\langle\varphi(u)-\varphi\left(w_{i}\right), v\right\rangle<\gamma\|v\|, \quad \forall u \in \mathcal{X}_{i} \forall v \in H .
$$


Thus, since the $\beta_{i}$ 's vanish outside of the $\mathcal{T}_{i}$ 's, we get

$$
\beta_{i}(u)\left\langle\varphi(u)-\varphi\left(w_{i}\right), v\right\rangle \leqslant \gamma \beta_{i}(u)\|v\| \quad \forall u, v \in H .
$$

If we sum the above expression over the $i$ 's, we get

$$
\left\langle\varphi(u)-\sum_{i} \beta_{i}(u) \varphi\left(w_{i}\right), v\right\rangle \leqslant \gamma\|v\|, \quad \forall u, v \in H .
$$

If we set $\tilde{\varphi}(u)=\Sigma_{i} \beta_{i}(u) \varphi\left(w_{i}\right)$, by the above formula we get

$$
\|\varphi(u)-\tilde{\varphi}(u)\| \leqslant \gamma, \quad \forall u \in H .
$$

Thus $\tilde{\varphi}$ satisfies (3-1)(c). To prove that $\tilde{\varphi}$ satisfies (3-1)(a) and (3-1)(b), observe that for each bounded ball $B_{R} \subset H$

$$
\tilde{\varphi}\left(B_{R}\right) \subset\left\{\text { convex hull of } \varphi\left(B_{R+1}\right)\right\} .
$$

Indeed, for $u \in B_{R}, \tilde{\varphi}(u)$ is a convex combination of elements $w_{i} \in B_{R+1}$. Since $\varphi\left(B_{R+1}\right)$ is relatively compact, so is its convex hull. Therefore $\tilde{\varphi}\left(B_{R}\right)$ is relatively compact and this proves (3-1)(a). Moreover $\tilde{\varphi}$ is locally Lipschitz continuous since in each point it is the finite sum of locally Lipschitz continuous functions. Thus also (3-1)(b) holds.

It remains to prove that $\tilde{\varphi}$ may be chosen $T_{g}$-equivariant whenever $\varphi$ is $T_{g}$-equivariant. Suppose we have constructed $\varphi_{1}$ which satisfies the (3-1) but it is not necessarily equivariant. We set

$$
\tilde{\varphi}(u)=\int_{G} T_{g}^{-1} \varphi_{1}\left(T_{g} u\right) d \mu
$$

where $\mu$ is the Haar measure on the group $G$. Clearly $\tilde{\varphi}$ is $T_{g}$-equivariant. We claim that it satisfies the (3-1). First of all, let us prove the compactness. For any $R>0$ we set $K=\overline{\varphi_{1}\left(T_{g} B_{R}\right)}=\overline{\varphi_{1}\left(B_{R}\right)}, \forall g \in G$. $\left(T_{g} B_{R}=B_{R}\right.$ because $T_{g}$ is unitary.) Clearly

$$
\tilde{\varphi}\left(B_{R}\right)=\int_{G} T_{g}^{-1} K d \mu=\left\{v=\int_{G} T_{g}^{-1} u d \mu \mid u \in K\right\} .
$$

Since the map $u \mapsto \int_{G} T_{g}^{-1} u d \mu$ is continuous, $\overline{\tilde{\varphi}\left(B_{R}\right)}$ is compact and this proves (3-1)(a). Now let us prove (3-1)(b). Take $u \in H$; the set $\left\{T_{g} u\right\}_{g \in G}$ is compact since $G$ is a compact group. Therefore by Lemma 3.1, there exist a neighborhood $\mathfrak{x}=$ $N_{d}\left(\left\{T_{g} u\right\}_{g \in G}\right)$ and a constant $l>0$ such that

$$
\left\|\varphi_{1}(v)-\varphi_{1}(w)\right\| \leqslant l\|v-w\| \quad \forall u, v \in \Re .
$$

For each $u \in \mathfrak{R}$ there exists a neighborhood $\mathfrak{R}_{0}$ such that $T_{g} v$ and $T_{g} w \in \mathfrak{X}$ whenever $v$ and $w \in \mathscr{T}_{0}$. Then for each $v$ and $w \in \mathscr{T}_{0}$ we have

$$
\begin{aligned}
\|\tilde{\varphi}(v)-\tilde{\varphi}(w)\| & =\left\|\int_{G} T_{g}^{-1}\left[\varphi_{1}\left(T_{g} v\right)-\varphi_{1}\left(T_{g} w\right)\right] d \mu\right\| \\
& \leqslant \int_{G}\left\|\varphi_{1}\left(T_{g}(v)\right)-\varphi_{1}\left(T_{g}(w)\right)\right\| d \mu \\
& \leqslant l \int_{G}\left\|T_{g}(v-w)\right\| d \mu \text { by }(3-2) \\
& =l\|v-w\| .
\end{aligned}
$$


Finally we prove (3-1)(c): using the fact that $\varphi$ is equivariant

$$
\begin{aligned}
\|\tilde{\varphi}(u)-\varphi(u)\| & =\left\|\int_{G} T_{g}^{-1} \varphi_{1}\left(T_{g} u\right) d \mu-\int_{G} T_{g}^{-1} \varphi\left(T_{g} u\right) d \mu\right\| \\
& =\left\|\int_{G} T_{g}^{-1}\left[\varphi_{1}\left(T_{g} u\right)-\varphi\left(T_{g} u\right)\right] d \mu\right\| \leqslant \int_{G}\left\|\varphi_{1}\left(T_{g} u\right)-\varphi\left(T_{g} u\right)\right\| \leqslant \gamma
\end{aligned}
$$

This completes the proof.

Lemma 3.3. Suppose that $L+\varphi \in \Gamma_{0}(H)$ and that the following Cauchy problem

$$
\left\{\begin{array}{l}
\dot{\eta}(t)=L \eta(t)+\varphi(\eta(t)), \\
\eta(0)=u
\end{array}\right.
$$

has a unique solution $\mathcal{Q}(t, u)\left(\mathcal{Q}(t, \cdot) \in \bigodot^{1}\left(\mathbf{R}, \bigodot^{0}(H, H)\right)\right)$. We suppose that for every $t, \mathcal{Q}(t, \cdot)$ maps bounded sets into bounded sets. Then for every $t \in \mathbf{R}$, Q $(t, u)$ has the following form

$$
\mathcal{Q}(t, u)=e^{t L} u-g(t, u)
$$

where $g(t, \cdot)$ is a compact operator for each $t \in \mathbf{R}$.

Proof. Without loss of generality, we suppose $t>0$. The operator $\mathscr{U}(t, u)$ satisfies the following identity which is the Volterra form of the equation (3-4):

$$
\mathcal{U}(t, u)=e^{t L} u+\int_{0}^{t} e^{(t-s) L} \varphi(\mathcal{Q}(s, u)) d s .
$$

We have to show that the operator

$$
g(t, u)=-\int_{0}^{t} e^{(t-s) L} \varphi(\mathscr{U}(s, u)) d s
$$

is compact. By our assumptions, for every $R>0$, there exists $\bar{R} \geqslant R$ such that

$$
\text { थ }\left(s, B_{R}\right) \subset B_{R}^{-} \quad \forall s \in[0, t]
$$

then

$$
\varphi\left(\mathscr{Q}\left(s, B_{R}\right)\right) \subset \varphi\left(B_{R}^{-}\right)
$$

where $\varphi\left(B_{R}\right)$ is a precompact subset of $H$. Now we set

$$
K=\overline{\left[\bigcup_{s \in[0, t]} e^{(t-s) L} \varphi\left(B_{R}^{-}\right)\right]} .
$$

$K$ is a compact subset of $H$. In fact let $\left\{v_{n}\right\}$ be a sequence in $K$; then there exist two sequences $\left\{s_{n}\right\} \subset[0, t]$ and $\left\{w_{n}\right\} \subset \varphi\left(B_{R}^{-}\right)$such that

$$
v_{n}=e^{\left(t-s_{n}\right) L} \varphi\left(w_{n}\right) \text {. }
$$

Now let $\left\{s_{n_{k}}\right\}$ and $\left\{w_{n_{k}}\right\}$ be two subsequences such that

$$
\left\{s_{n_{k}}\right\} \rightarrow \bar{s} \text { and } \varphi\left(w_{n_{k}}\right) \rightarrow \bar{z} \in \overline{\varphi\left(B_{R}\right)}
$$

then

$$
v_{n_{k}} \rightarrow e^{(t-\bar{s}) L} \bar{z} \in K
$$

Therefore $K$ is compact. Now set

$$
K_{1}=\overline{\{\text { convex hull of } K\}} \text {. }
$$


By a well-known theorem $K_{1}$ is compact. Since for every $u \in B_{R}^{-}$,

$$
\int e^{(t-s) L} \varphi(\mathscr{Q}(s, u)) d s \in K_{1}
$$

$-g(t, u) \in K$. Therefore $g(t, \cdot)$ is compact.

TheOREM 3.4 (DEFORMATION THEOREM). Let $f \in \mathbb{C}^{1}(H)$ be a function which satisfies the following assumptions

$\left(\mathrm{f}_{1}\right) f(u)=\frac{1}{2}\langle L u, u\rangle+\Phi(u)$ where $L$ is a bounded selfadjoint operator and $\varphi^{\text {def }}=\Phi^{\prime}$ is compact.

$\left(\mathrm{f}_{2}\right) f$ satisfies P.S. in $\left[c_{0}, c_{\infty}\right]$ i.e. any sequence $\left\{u_{m}\right\} \subset H$ such that $f\left(u_{m}\right) \rightarrow c \in$ $\left[c_{0}, c_{\infty}\right]$ and $f^{\prime}\left(u_{m}\right) \rightarrow 0$ as $m \rightarrow+\infty$, has a convergent subsequence.

Then $\forall c \in\left[c_{0}, c_{\infty}\right], \forall \Re=N_{d}\left(\mathbf{K}_{c}\right), \exists \varepsilon>0$ and $\exists \eta=\bar{e}^{\delta L}+\psi$ (where $\delta>0$ is $a$ constant and $\psi$ a compact operator) such that

$$
\eta\left(\Theta_{c+\varepsilon} \backslash \Re\right) \subset Q_{c-\varepsilon}
$$

where $\mathbf{K}_{c}, \mathbb{Q}_{c}$ are defined by (1.8).

Moreover, if

$\left(\mathrm{f}_{3}\right) f$ is $T_{g}$-invariant

then $\eta$ is $T_{g}$-equivariant, i.e. $\eta \in \Re^{*}$.

Proof. Because of $\left(\mathrm{f}_{2}\right), \mathbf{K}_{c}$ is a compact set. Then $\mathscr{N}$ is bounded. Let $\rho_{1}>0$ be a constant such that $\mathfrak{R} \subset B_{\rho_{1}}$. We set

$$
\delta=\frac{d}{4\left(1+2\|L\| \rho_{1}\right)} .
$$

Because of $\left(\mathrm{f}_{2}\right)$, there exists $\varepsilon>0$ such that

$$
\left\|f^{\prime}(u)\right\| \geqslant \sqrt{\frac{4 \varepsilon}{\delta}} \text { for } u \in Z_{c, \varepsilon} \backslash \Re_{d / 2}
$$

where $Z_{c, \varepsilon}=f^{-1}([c-\varepsilon, c+\varepsilon])$ and $\Re_{d / 2}=N_{d / 2}\left(\mathbf{K}_{c}\right)$.

In fact suppose that (3-7) does not hold. Then there exist a sequence $\left\{u_{n}\right\} \subset H$ and a sequence $\varepsilon_{n} \downarrow 0$ such that $u_{n} \in Z_{c, \varepsilon_{n}} \backslash \mathcal{X}_{d / 2}$ and $\left\|f^{\prime}\left(u_{n}\right)\right\| \leqslant \sqrt{4 \varepsilon_{n} / \delta}$. Thus we have that $f\left(u_{n}\right) \rightarrow c$ and $\left\|f^{\prime}\left(u_{n}\right)\right\| \rightarrow 0$, and by $\left(\mathrm{f}_{2}\right),\left\{u_{n}\right\}$ converges to some point $\bar{u}$. Then we have that $f(\bar{u})=c$ and $f^{\prime}(\bar{u})=0$ and this contradicts the fact that $\bar{u} \notin \mathbf{K}_{c}$. Thus (3-7) holds. Also we can suppose that

$$
\varepsilon \leqslant \delta / 5 \text {. }
$$

We now set

$$
\gamma=\min \left(\varepsilon / 2 \delta ; \frac{1}{2} \sqrt{\varepsilon / 2 \delta}\right) .
$$

Now, by virtue of Lemma 3.2, there exists a compact, locally Lipschitz continuous operator $\tilde{\varphi}$ such that

$$
\|\varphi(u)-\tilde{\varphi}(u)\| \leqslant \gamma, \quad \forall u \in H .
$$

By the above inequality we get

$$
\|\varphi(u)\|^{2}-2\langle\varphi(u), \tilde{\varphi}(u)\rangle+\|\tilde{\varphi}(u)\|^{2} \leqslant \gamma^{2} .
$$


Then

$$
\begin{aligned}
\langle\varphi(u), \tilde{\varphi}(u)\rangle & \geqslant \frac{1}{2}\left(\|\varphi(u)\|^{2}+\|\tilde{\varphi}(u)\|^{2}\right)-\gamma^{2} / 2 \\
& \geqslant\|\varphi(u)\| \cdot\|\tilde{\varphi}(u)\|-\gamma^{2} / 2 .
\end{aligned}
$$

Now set $\chi(t)=\min (1,1 / t)$ and

$$
V(u)=\chi\left(\frac{\|\tilde{\varphi}(u)\|}{1+\|L u\|}\right) \tilde{\varphi}(u)
$$

It is easy to check that $V$ is a compact, locally Lipschitz continuous operator and that

$$
\|V(u)\| \leqslant 1+\|L u\|, \quad \forall u \in H
$$

We now claim that

$$
\left\langle f^{\prime}(u), L u+V(u)\right\rangle \geqslant \frac{3 \varepsilon}{\delta} \quad \forall u \in Z_{c, \varepsilon} \backslash \Re_{d / 2} .
$$

In order to prove (3-13) we distinguish two cases.

First case. Suppose that $\|\tilde{\varphi}(u)\| \leqslant 1+\|L(u)\|$. Then we have

$$
\begin{aligned}
\left\langle f^{\prime}(u), L u+V(u)\right\rangle & =\left\langle f^{\prime}(u), L u+\tilde{\varphi}(u)\right\rangle \\
& =\left\langle f^{\prime}(u), L u+\varphi(u)\right\rangle-\left\langle f^{\prime}(u), \varphi(u)-\tilde{\varphi}(u)\right\rangle \\
& \geqslant\left\|f^{\prime}(u)\right\|^{2}-\gamma\left\|f^{\prime}(u)\right\| \quad \text { by }(3-10) \\
& \geqslant 3 \varepsilon / \delta \quad \text { by }(3-7) \text { and (3-9). }
\end{aligned}
$$

Second case. Suppose that

$$
\|\tilde{\varphi}(u)\| \geqslant 1+\|L(u)\| .
$$

By the definition of $V$ we get

$$
\begin{aligned}
\left\langle f^{\prime}(u), L u+V(u)\right\rangle= & \left\langle L u+\varphi(u), L u+\frac{1+\|L u\|}{\|\tilde{\varphi}(u)\|} \tilde{\varphi}(u)\right\rangle \\
= & \|L u\|^{2}+\frac{1+\|L u\|}{\|\tilde{\varphi}(u)\|}\langle L u, \tilde{\varphi}(u)\rangle+\langle\varphi(u), L u\rangle \\
& +\frac{1+\|L u\|}{\|\tilde{\varphi}(u)\|}\langle\varphi(u), \tilde{\varphi}(u)\rangle \\
\geqslant & \|L u\|^{2}-(1+\|L u\|) \cdot\|L u\|-\|L u\| \cdot\|\varphi(u)\| \\
& +(1+\|L u\|)\|\varphi(u)\|-\frac{1+\|L u\|}{\|\tilde{\varphi}(u)\|} \cdot \frac{\gamma^{2}}{2} \quad \text { by }(3-11) \\
\geqslant & \|L u\|^{2}-\|L u\|-\|L u\|^{2}-\|L u\| \cdot\|\varphi(u)\| \\
& +\|\varphi(u)\| \cdot\|L u\|+\|\varphi(u)\|-\frac{\gamma^{2}}{2} \quad \text { by }(3-14) \\
\geqslant & \|\varphi(u)\|-\|L u\|-\frac{\gamma^{2}}{2} \\
\geqslant & \|\tilde{\varphi}(u)\|-\gamma-\|L u\|-\gamma^{2} / 2 \quad \text { by (3-10) } \\
\geqslant & 1-\gamma-\gamma^{2} / 2 \quad \text { by }(3-14) \\
\geqslant & 5 \varepsilon / \delta-\varepsilon / 2 \delta-1 / 4 \cdot \varepsilon / 2 \delta \geqslant 3 \varepsilon / \delta \quad \text { by (3-8) and (3-9). } \\
&
\end{aligned}
$$

Then (3-13) is proved. 
Now consider the following Cauchy problem in the Hilbert space $H$

$$
\left\{\begin{array}{l}
\frac{1}{d t} \xi(t)=-L \xi(t)-V(\xi(t)) \\
\xi(0)=u
\end{array}\right.
$$

Since $V$ is locally Lipschitz continuous, (3-15) has a unique local solution. Moreover, by $(3-12)$, we have that

$$
\|L u+V(u)\| \leqslant 1+2\|L\| \cdot\|u\| .
$$

Then by standard results on O.D.E.'s it follows that $\xi(t)$ is defined for all $t \in \mathbf{R}$.

We shall denote by $\mathcal{Q}(t, u)$ the flow relative to the Cauchy problem (3-15). Now we shall prove that

$$
\mathcal{Q}(t, u) \in H \backslash \Re_{d / 2} \text { for each } u \in H \backslash \Re_{d} \text { and for each } t \in[0, \delta] .
$$

In order to prove this we argue indirectly.

Suppose that there are points $t_{0}, t_{1} \in[0, \delta]\left(t_{0}<t_{1}\right)$ such that

$$
\mathscr{U}\left(t_{0}, u\right) \in \partial \mathscr{N}_{d} ; \mathscr{U}\left(t_{1}, u\right) \in \partial \mathscr{N}_{d / 2} \text { and } \mathscr{U}(t, u) \in \overline{\mathscr{N}_{d} \backslash \mathscr{N}_{d / 2}}
$$

for all $t \in\left[t_{0}, t_{1}\right]$.Then we have

$$
\begin{aligned}
d / 2 & \leqslant\left\|\mathscr{Q}\left(t_{1}, u\right)-\mathscr{U}\left(t_{0}, u\right)\right\|=\left\|\int_{t_{0}}^{t_{1}} \frac{d}{d t} \mathscr{Q}(t, u) d t\right\| \\
& =\left\|\int_{t_{0}}^{t_{1}}\{-L \mathscr{Q}(t, u)-V(\mathcal{Q}(t, u))\} d t\right\| \\
& \leqslant \int_{t_{0}}^{t_{1}}\|L \mathscr{Q}(t, u)+V(\mathcal{Q}(t, u))\| d t \\
& \leqslant \int_{t_{0}}^{t_{1}}(1+2\|L\|\|\mathscr{Q}(t, u)\|) d t \quad \text { by }(3-16) \\
& \leqslant\left(1+2\|L\| \rho_{1}\right)\left(t_{1}-t_{0}\right) \leqslant\left(1+2\|L\| \rho_{1}\right) \delta .
\end{aligned}
$$

The last inequalities have been possible since $\mathfrak{T}_{d} \subset B_{\rho_{1}}$. Then using the definition of $\delta$ we get

$$
d / 2 \leqslant \frac{d}{4\left(1+2\|L\| \rho_{1}\right)} \cdot\left(1+2\|L\| \rho_{1}\right)=d / 4 .
$$

This is a contradiction, then (3-17) is proved.

Next we shall prove that

$$
\text { Q }(\delta, u) \in \mathbb{Q}_{c-\varepsilon}, \quad \forall u \in \mathbb{U}_{c+\varepsilon} \backslash \Re_{d} .
$$

First of all, we remark that, by virtue of (3-13), we have

$$
\frac{d}{d t} f(\mathscr{\vartheta}(t, u))=\left\langle f^{\prime}(\mathscr{Q}(t, u)), \frac{d}{d t} \text { थ }(t, u)\right\rangle \leqslant-3 \varepsilon / \delta \text {. }
$$

In order to prove (3-18), we have to show that

$$
\begin{aligned}
& \text { there exists } t_{0} \in[0, \delta] \text { such that } f\left(\mathscr{U}\left(t_{0}, u\right)\right) \leqslant c-\varepsilon \\
& \forall u \in \mathscr{Q}_{c+\varepsilon} \backslash \mathscr{X}_{d}
\end{aligned}
$$


and

$$
\begin{aligned}
& \text { if } \exists t_{1} \in \mathbf{R} \text { such that } f\left(\mathcal{Q}\left(t_{1}, u\right)\right)=c-\varepsilon, \text { then } f(\mathscr{Q}(t, u)) \\
& \leqslant c-\varepsilon \quad \forall t \geqslant t_{1} .
\end{aligned}
$$

First we prove (3-20). We argue indirectly and we suppose that for each $t \in[0, \delta]$, $f(\mathcal{Q}(t, u))>c-\varepsilon$ then we have

$$
2 \varepsilon \geqslant-[f(\mathscr{U}(\delta, u))-f(\mathscr{U}(0, u))]=-\int_{0}^{\delta} \frac{d}{d t} f(\mathscr{U}(t, u)) d t .
$$

By (3-17), $\mathscr{Q}(t, u) \notin \mathscr{T}_{d / 2}$. Moreover, by (3-19), the function $t \mapsto f(\mathcal{Q}(t, u))$ is decreasing. Then we have that $\mathscr{Q}(t, u) \in Z_{c, \varepsilon} \backslash \Re_{d / 2}$. Therefore, using again (3-19) and by (3-22), it follows that

$$
2 \varepsilon \geqslant \int_{0}^{\delta}(3 \varepsilon / \delta) d t=3 \varepsilon
$$

This is a contradiction, thus (3-20) is proved. (3-21) follows directly from (3-19). Then (3-18) is proved. By Lemma 3.3, we have

$$
\text { थ }(t, u)=e^{-t L}+g(t, u)
$$

where $g(t, \cdot)$ is a compact operator for each $t \in \mathbf{R}$.

If we set

$$
\eta(u)=\mathscr{Q}(\delta, u)
$$

we see that $\eta$ has the desired form with $\psi(u)=g(\delta, u)$. Moreover, by (3-18), (3-6) follows. Then the first part of the theorem is proved.

If $f$ is $T_{g}$-invariant, then $L$ and $\varphi$ are $T_{g}$-equivariant. Then by the last part of Lemma $3.2, \tilde{\varphi}$ can be chosen equivariant. Since $T_{g}$ is a unitary representation, the functional

$$
\chi\left(\frac{\|\tilde{\varphi}(u)\|}{1+\|L u\|}\right)
$$

is $T_{g}$-invariant. In fact

$$
\chi\left(\frac{\left\|\tilde{\varphi}\left(T_{g} u\right)\right\|}{1+\left\|L T_{g} u\right\|}\right)=\chi\left(\frac{\left\|T_{g} \tilde{\varphi}(u)\right\|}{1+\left\|T_{g} L u\right\|}\right)=\chi\left(\frac{\|\tilde{\varphi}(u)\|}{1+\|L u\|}\right) .
$$

Then $V$ is a $T_{g}$-equivariant operator. Therefore the operator $\mathscr{Q}(t, \cdot)$ and consequently $\eta$ are $T_{g}$-equivariant.

By the deformation theorem the following corollary follows.

COROLlaRY 3.5. If $f$ satisfies $\left(\mathrm{f}_{1}\right),\left(\mathrm{f}_{2}\right)$ and $\left(\mathrm{f}_{3}\right)$ then $\left\{f, c_{0}, c_{\infty}\right\}$ satisfy the property (P) with respect to $\left\{\Sigma, \Re^{*}\right\}$.

Proof. By $\left(\mathrm{f}_{3}\right)$ it follows that $\Theta_{c}$ and $\mathbf{K}_{c} \in \Sigma$. By $\left(\mathrm{f}_{2}\right)$, it follows that $\mathbf{K}_{c}$ is compact. Then (1-9)(a) is satisfied. (1-9)(b) follows directly by Theorem 3.4 .

4. Some abstract multiplicity theorems. In this section we shall use the theory developed in the previous sections to give a lower bound for the number of critical values of the functional $(0.1)$ in several different situations. 
THEOREM 4.1. Let $H$ be a Hilbert space with an index theory I related to a representation $T_{g}$ which satisfies the dimension property.

Let $f$ be a functional which satisfies $\left(\mathrm{f}_{1}\right)\left(\mathrm{f}_{2}\right)\left(\mathrm{f}_{3}\right)$ of Theorem 3.4. Moreover suppose that there are two invariant spaces $H^{+}, H^{-} \in \Sigma$ and a constant $\rho>0$ such that

$$
\begin{cases}\text { (a) } F \subset H^{+}, & L H^{+}=H^{+}, \\ \text {(b) } f(u)>c_{0} & \forall u \in H^{+}, \\ \text {(c) } f(u)<c_{\infty} & \forall u \in H^{-} \cap S_{\rho} .\end{cases}
$$

Then, if $I_{1}^{*}$ is the pseudoindex theory (2-7) and if the integer

$$
\bar{k}=\frac{1}{d}\left[\operatorname{dim}\left(H^{+} \cap H^{-}\right)-\operatorname{cod}\left(H^{+}+H^{-}\right)\right],
$$

is well defined and positive, the numbers

$$
c_{k}=\inf _{i_{1}^{*}(A) \geqslant k} \sup _{u \in A} f(u) \text { for } k=1, \ldots, \bar{k}
$$

are critical values of $f$ and $c_{0} \leqslant c_{1} \leqslant \cdots \leqslant c_{k}^{-} \leqslant c_{\infty}$. Moreover if

$$
\left\{\begin{array}{l}
c=c_{k}=\cdots=c_{k+r} \\
\text { then } \\
i\left(\mathbf{K}_{c}\right) \geqslant r+1 .
\end{array}\right.
$$

Proof. We apply Theorem 1.4. By $\left(\mathrm{f}_{1}\right)\left(\mathrm{f}_{2}\right)\left(\mathrm{f}_{3}\right)$ and Corollary 3.5 , the triplet $\left\{f, c_{0}, c_{\infty}\right\}$ satisfies the property (P). Now take $A \in \Sigma$ with $A \subset \mathbb{Q}_{c_{0}}$; then by $\left(\mathrm{f}_{4}\right)(\mathrm{b})$, $A \cap H^{+}=\varnothing$. Therefore $i_{1}^{*}(A)=i\left(A \cap H^{+}\right)=0$ and (1-10)(b) is satisfied. Setting $\tilde{A}=S_{\rho} \cap H^{-}$, by $\left(\mathrm{f}_{3}\right)(\mathrm{c})$ we have $\tilde{A} \subset \mathbb{Q}_{c_{\infty}}$, then by Theorem $2.4 i_{1}^{*}(\tilde{A})=\bar{k}$. This shows that (1-10)(c) holds and the conclusion follows.

We now give a theorem analogous to Theorem 4.1 which makes use of the pseudoindex $I_{2}^{*}$.

THEOREM 4.2. Suppose that all the assumptions of Theorem 4.1, but $\left(\mathrm{f}_{4}\right)$ are satisfied. Instead suppose that

$$
\begin{cases}\text { (a) } F \cap H^{+}=\{0\} ; & F \subset H^{-}, L H^{-}=H^{-}, \\ \text {(b) } f(u)>c_{0} & \forall u \in H^{+} \cap S_{\rho}, \\ \text { (c) } f(u)<c_{\infty} & \forall u \in H^{-} .\end{cases}
$$

Then, if $I_{2}^{*}$ is the pseudoindex theory (2-8), and $\vec{k}$ is well defined by (4-1) and positive, the numbers

$$
c_{k}=\inf _{i_{2}^{*}(A) \geqslant k} \sup _{u \in A} f(u)
$$

are critical values of $f$ and $c_{0} \leqslant c_{1} \leqslant \cdots \leqslant c_{k} \leqslant c_{\infty}$. Moreover (4-2) holds.

Proof. We apply again Theorem 1.4. By $\left(\mathrm{f}_{1}\right),\left(\mathrm{f}_{2}\right)$ and $\left(\mathrm{f}_{3}\right)$ and Corollary 3.5 , the triplet $\left\{f, c_{0}, c_{\infty}\right\}$ satisfies the property $(\mathrm{P})$.

Now take $A \in \Sigma$ such that $A \subset \mathbb{Q}_{c_{0}}$; then by (f $\left.\mathrm{f}_{4}^{*}\right)(\mathrm{b}), A \cap H^{+} \cap S_{\rho}=\varnothing$. Therefore $\min _{h \in \mathscr{O}^{*}} i\left(h(A) \cap H^{+} \cap S_{\rho}\right)=0$ and (1-10)(b) is satisfied. Moreover, by (f $\left.\mathrm{f}_{4}^{*}\right)(\mathrm{a})$, the assumptions of Theorem 2.5 are satisfied. Then $i_{2}^{*}\left(H^{-}\right)=\bar{k}$. 
Therefore setting $\tilde{A}=H^{-},(1-10)($ c) follows.

REMARK 4.3. If $f$ satisfies the assumptions of Theorem 4.1, thus $-f$ essentially satisfies the assumptions of Theorem 4.2 if we interchange $\mathrm{H}^{+}$and $\mathrm{H}^{-}$, and $c_{0}$ and $c_{\infty}$.

Those two theorems are related to Theorem 2.19 of [3] and Theorem 12 of [8] for indefinite functionals. Actually in [3] and in [8] only even functionals have been considered, and of course $\left(f_{1}\right)$ is not required since the functionals are semidefinite. Even if those theorems sound different, in a deeper analysis the similarity is evident (also cf. Theorem 2.19 of [3] with Corollary 4.5). While Theorems 2.19 of [3] and 12 of [8] apply to two completely different classes of problems we see that in Theorems 4.1 and 4.2, those two different situations are unified.

REMARK 4.4. Theorems 4.1 and 4.2 are not sufficient to guarantee the existence of at least $\bar{k}$ (orbits of) critical points. In fact it might happen that $\mathbf{K}_{c} \cap F \neq \varnothing$. Then $i\left(\mathbf{K}_{c}\right)=+\infty$ but it might contain only one fixed point (cf. (2.3) and the following remarks). Thus to have a multiplicity result we must add some assumptions depending on the nature of $F$ and $f$. For example in Theorem 4.1, we could assume

$$
\inf _{u \in F} f(u)=\nu>c_{\infty}
$$

and this, of course, implies $\mathbf{K}_{c} \cap F=\varnothing$ and the multiplicity result. A similar argument is used in the proof of Theorem 0.1 given below and Theorems 5.1 and 5.3. More subtle arguments for proving $K_{c} \cap F=\varnothing$ are given in the applications to Hamiltonian systems in $§ 5$.

Proof of TheOrem 0.1. We shall use Theorem 4.1 (but we could use as well Theorem 4.2).

$\left(\bar{f}_{1}\right)$ is equal to $\left(f_{1}\right)$ and $\left(\bar{f}_{2}\right)$ implies $\left(f_{2}\right)$.

$\left(\bar{f}_{3}\right)$ implies $\left(f_{3}\right)$ where the group representation is the one described in Example I of $\S 2$.

For such representation $F=\{0\}$, thus $\left(\mathrm{f}_{4}\right)(\mathrm{a})$ is satisfied.

$\left(\mathrm{f}_{4}\right)(\mathrm{b})$ and $(\mathrm{c})$ follow by $\left(\overline{\mathrm{f}}_{4}\right)$.

Thus Theorem 4.1 can be applied.

Then in order to get the conclusion of Theorem 0.1 , it is necessary to prove that $K_{c} \cap F=\varnothing$ but this follows from Remark 4.4.

By Theorem 4.2, the following corollary follows.

COROLlary 4.5. Suppose that $f$ satisfies all the assumptions of Theorem 4.2, except $\left(\mathrm{f}_{4}^{*}\right)(\mathrm{c})$. Moreover suppose that

$$
\begin{cases}\text { (a) } & \operatorname{dim}\left(H^{+}\right)^{\perp}=+\infty \\ \text { (b) } & f(u) \text { is upper bounded on }\left(H^{+}\right)^{\perp} \oplus V^{d k} \\ & \text { for every } V^{d k} \in \Sigma, V^{d k} \subset H^{+} .\end{cases}
$$

Then (4-3) defines a critical value for each $k \in \mathbf{N}, c_{k} \leqslant c_{k+1}$, and (4-2) holds.

Proof. It is enough to choose $\bar{k} \in \mathbf{N}$ arbitrarily and to show that the $c_{k}$ 's are critical values for $k \leqslant \bar{k}$. In order to do this, we set $H^{-}=\left(H^{+}\right)^{\perp} \oplus V^{d \bar{k}}$ and apply Theorem 4.2. 
From Theorem 4.1, we shall deduce another theorem which is less general, but it can be applied more directly to some concrete problems we shall consider later. First of all we make the following assumption on $L$ :

$0 \notin \sigma_{e}(L)$ where $\sigma_{e}(L)$ denotes the essential spectrum of $L$ $\left(\mathrm{f}_{5}\right) \quad$ (this means that 0 is either an isolated eigenvalue of finite multiplicity or it belongs to the resolvent).

Then we can decompose $H$ in the following way

$$
H=V^{-} \oplus V^{0} \oplus V^{+}
$$

where

$$
\begin{aligned}
& V^{0}=\operatorname{ker} L \\
& \langle L u, u\rangle \leqslant \alpha\|u\|^{2} \quad \forall u \in V^{-}, \\
& \langle L u, u\rangle \geqslant \beta\|u\|^{2} \quad \forall u \in V^{+},
\end{aligned}
$$

where $\alpha<0 \leqslant \beta$ are suitable constants.

If $Q$ is a compact operator, by well-known theorems, (see e.g. [17]) $\sigma_{e}(L)=$ $\sigma_{e}(L+Q)$. Therefore $H$ has the following decomposition:

$$
H=W^{-} \oplus W^{0} \oplus W^{+}
$$

where

$$
\begin{aligned}
& W^{0}=\operatorname{ker}(L+Q), \\
& \langle(L+Q) u, u\rangle \leqslant \alpha^{\prime}\|u\|^{2} \quad \forall u \in W^{-}, \\
& \langle(L+Q) u, u\rangle \geqslant \beta^{\prime}\|u\|^{2} \quad \forall u \in W^{+},
\end{aligned}
$$

where $\alpha^{\prime}<0<\beta^{\prime}$ are suitable constants.

The following theorem holds.

THEOREM 4.6. Suppose that $f$ satisfies $\left(\mathrm{f}_{1}\right),\left(\mathrm{f}_{3}\right),\left(\mathrm{f}_{5}\right)$ and

$$
\begin{cases}\text { (a) } & \Phi(0)=0 . \\ \text { (b) } & \text { every sequence }\left\{u_{n}\right\} \text { such that } f\left(u_{n}\right) \rightarrow c<0 \text { and } \\ & \left\|f^{\prime}\left(u_{n}\right)\right\| \rightarrow 0 \text { as } n \rightarrow+\infty \text { is bounded. } \\ \text { (c) } & \text { there exists a compact operator } Q \text { such that } \\ & \varphi(u)=Q u+o(\|u\|) \text { where } \varphi=\Phi^{\prime} . \\ \text { (d) } & \lim _{\| u \rightarrow+\infty} \frac{\|\varphi(u)\|}{\|u\|}=0 . \\ \text { (e) } & F \subset V^{+} .\end{cases}
$$

If we set $H^{+}=V^{+}$and $H^{-}=W^{-}$, then the same conclusion of Theorem 4.1 holds with $c_{0}$ and $c_{\infty}$ suitable negative constants. In particular we have

$$
\begin{aligned}
\bar{k} & =\frac{1}{d} \operatorname{dim}\left(V^{+} \cap W^{-}\right)-\frac{1}{d} \operatorname{cod}\left(V^{+}+W^{-}\right) \\
& =\frac{1}{d} \operatorname{dim}\left(V^{+} \cap W^{-}\right)-\frac{1}{d} \operatorname{dim}\left[\left(V^{-} \oplus V^{0}\right) \cap\left(W^{+} \oplus W^{0}\right)\right]
\end{aligned}
$$

where the above spaces are defined by (4-4) and (4-4'). 
REMARK 4.7. If $L$ has discrete spectrum and it commutes with $Q$, then $\bar{k}$ is easy to compute.

In fact, let $\left\{e_{k}\right\}_{k \in \mathbf{N}}$ be a common set of eigenvectors of $L$ and $Q$ corresponding to the eigenvalues $\left\{\lambda_{k}\right\}$ of $L$ and $\left\{q_{k}\right\}$ of $Q$.

Then

$$
\begin{aligned}
V^{+} \cap W^{-} & =\operatorname{Sp}\left\{e_{k} \mid \lambda_{k}>0 ; \lambda_{k}+q_{k}<0\right\} \\
& =\operatorname{Sp}\left\{e_{k} \mid 0<\lambda_{k}<-q_{k}\right\}
\end{aligned}
$$

and

$$
\begin{aligned}
\left(V^{-} \oplus V^{0}\right) \cap\left(W^{+} \oplus W^{0}\right) & =\operatorname{Sp}\left\{e_{k} \mid \lambda_{k} \leqslant 0 ; \lambda_{k}+q_{k} \geqslant 0\right\} \\
& =\operatorname{Sp}\left\{e_{k} \mid 0 \geqslant \lambda_{k} \geqslant-q_{k}\right\} .
\end{aligned}
$$

Therefore

$$
\bar{k}=\#\left\{k \in \mathbf{Z} \mid 0<\lambda_{k}<-q_{k}\right\}-\#\left\{k \in \mathbf{Z} \mid 0 \geqslant \lambda_{k} \geqslant-q_{k}\right\} .
$$

Before proving the Theorem 4.6 we need the following lemma.

LEMma 4.8. If $f$ satisfies $\left(\mathrm{f}_{1}\right),\left(\mathrm{f}_{5}\right)$ and $(4-5)(\mathrm{b})$ then it satisfies $\left(\mathrm{f}_{2}\right)$ in $\left[c_{0}, c_{\infty}\right]$ $\left(c_{0}<c_{\infty}\right)$.

Proof. Let $\left\{u_{n}\right\}$ be a sequence such that $f\left(u_{n}\right) \rightarrow c$ and

$$
f^{\prime}\left(u_{n}\right) \rightarrow 0 \text {. }
$$

By (4-5)(b) it is bounded.

By $\left(\mathrm{f}_{5}\right)$ there is a compact operator $K_{0}$ such that

$$
0 \notin \sigma\left(L+K_{0}\right) \text {. }
$$

By (4-6) we have that the sequence

$$
v_{n} \stackrel{\text { def }}{=}\left(L+K_{0}\right) u_{n}-K_{0} u_{n}+\varphi\left(u_{n}\right)
$$

is converging to 0 .

Since $\left\{u_{n}\right\}$ is bounded, $-K_{0} u_{n}+\varphi\left(u_{n}\right)$ is pre-compact, then the set

$$
D=\overline{\left\{K_{0} u_{n}-\varphi\left(u_{n}\right)+v_{n} \mid n \in \mathbf{N}\right\}}
$$

is compact.

By (4-7), $\left(L+K_{0}\right) u_{n} \in D$, then $u_{n} \in\left(L+K_{0}\right)^{-1} D$. Since $\left(L+K_{0}\right)^{-1} D$ is a compact set, $\left\{u_{n}\right\}$ has a converging subsequence.

ProOF OF THEOREM 4.6. We shall check all the assumptions of Theorem 4.1 with $H^{+}=V^{+}, H^{-}=W^{-}, c_{\infty}=\gamma_{1} / 2$ and $c_{0}=\gamma_{2}-1$ where $\gamma_{2}<\gamma_{1}<0$ are constants to be determined later. Because of Lemma 4.8, $\left(\mathrm{f}_{2}\right)$ holds. Then $\left(\mathrm{f}_{1}\right)\left(\mathrm{f}_{2}\right)$ and $\left(\mathrm{f}_{3}\right)$ are satisfied. Let us prove $\left(f_{4}\right)$. $\left(f_{4}\right)(a)$ follows by $(4.5)(e)$. Before proving $\left(f_{4}\right)(b)$, we shall prove $\left(\mathrm{f}_{4}\right)(\mathrm{c})$. We use the Taylor formula for $f$ at 0 :

$$
f(u)=\frac{1}{2}\langle L u, u\rangle+\frac{1}{2}\langle Q u, u\rangle+o\left(\|u\|^{2}\right) \text { for }\|u\| \rightarrow 0 .
$$

Then for $u \in W^{-}$we have $f(u) \leqslant \alpha^{\prime}\|u\|^{2}+o\left(\|u\|^{2}\right)$ where $\alpha^{\prime}$ is the constant appearing in (4-4'). Since $\alpha^{\prime}<0$, there exists a constant $\rho>0$ and $\gamma_{1}<0$ such that

$$
f(u)<\gamma_{1} \quad \forall u \in W^{-} \cap S_{\rho} .
$$

Setting $c_{\infty}=\gamma_{1} / 2,\left(\mathrm{f}_{4}\right)(\mathrm{c})$ is satisfied. 
In order to prove $\left(f_{4}\right)(b)$ we first observe that, by virtue of (4-5)(d), there is $r>0$ such that

$$
\|\varphi(u)\| \leqslant(\beta / 2)\|u\|^{2} \quad \text { whenever }\|u\|>r
$$

where $\beta$ is the constant appearing in (4-4).

Moreover, since $\varphi$ is compact, there is $M>0$ such that

$$
\|\varphi(u)\| \leqslant M \quad \text { if }\|u\| \leqslant r
$$

thus

$$
\|\varphi(u)\| \leqslant M+\beta / 2\|u\| \quad \forall u \in H .
$$

Using the above formula we get

$$
\begin{aligned}
|\Phi(u)| & =\left|\int_{0}^{1}\langle\varphi(t u), u\rangle d t\right| \\
& \leqslant\left|\int_{0}^{1}\left\{M \cdot\|u\|+\frac{\beta t}{2}\|u\|^{2}\right\} d t\right| \\
& \leqslant M\|u\|+\frac{1}{4} \beta\|u\|^{2} \quad \forall u \in H .
\end{aligned}
$$

Then by (4-4) and the above inequality, for every $u \in V^{+}$, we get

$$
\begin{aligned}
f(u) & =\frac{1}{2}\langle L u, u\rangle+\Phi(u) \\
& \geqslant(\beta / 2)\|u\|^{2}-|\Phi(u)| \geqslant(\beta / 4)\|u\|^{2}-M \cdot\|u\| .
\end{aligned}
$$

This implies that $f$ is bounded from below on $V^{+}$and we can set

$$
\gamma_{2}=\inf _{u \in V^{+}} f(u) \text { and } c_{0}=\gamma_{2}-w \text { with } w>0 \text { such that } \gamma_{2}-w<c_{\infty} .
$$

Then by Theorem 4.1 the conclusion follows.

REMARK 4.9. Under the assumptions of Theorem 4.6, we could equally well have used the pseudoindex theory $I_{2}^{*}$ and Theorem 4.2 in order to get similar results.

REMARK 4.10. If we strengthen $\left(f_{5}\right)$ in the following way

$$
0 \notin \sigma(L)
$$

then, by (4-5)(d), we can easily deduce (4-5)(b).

$\left(f_{5}^{*}\right)$ is a nonresonance condition at $\infty$.

Using this remark we have the following corollary.

Corollary 4.11. If $f$ satisfies $\left(\mathrm{f}_{1}\right),\left(\mathrm{f}_{3}\right),\left(\mathrm{f}_{5}^{*}\right),(4-5)(\mathrm{a})(\mathrm{c})(\mathrm{d})(\mathrm{e})$, then the same conclusion of Theorem 4.6 holds.

REMARK 4.12. If $\left(f_{4}\right)(a)$ (or equivalently $\left(f_{4}^{*}\right)(a)$ or $(4-5)(c)$ ) does not hold, then the pseudoindex theories studied in $\$ 2$ cannot be applied directly, and a general way to deal with such problems has not yet been developed. However, in [6], a method has been introduced which permits us to treat semidefinite functionals even when $\left(f_{4}\right)(a)$ does not hold (cf. Remark 6.9).

5. Applications to asymptotic linear Hamiltonian systems. Let $H \in \bigcup^{2}\left(\mathbf{R}^{2 n}, \mathbf{R}\right)$ and consider the Hamiltonian system of ordinary differential equations

$$
\dot{p}=-\frac{\partial H}{\partial q}(p, q), \quad \dot{q}=\frac{\partial H}{\partial p}(p, q),
$$


where $p$ and $q$ are $n$-tuples and denotes $d / d t$. Setting $z=(p, q)$ and $J=\left(\begin{array}{cc}0 & -\mathrm{Id} \\ \mathrm{Id} & 0\end{array}\right)$ where Id denotes the identity matrix in $\mathbf{R}^{n},(6-1)$ becomes

$$
\dot{z}=J H_{z}(z)
$$

where $H_{z}=\partial H / \partial z$. We are concerned in periodic solutions of fixed period of (5-2). We suppose that $H$ satisfies the following properties

$$
\begin{array}{lll}
\left(\mathrm{H}_{1}\right) & H_{z}(z)=A z+o(|z|) & \text { for } z \rightarrow+\infty, \\
\left(\mathrm{H}_{2}\right) & H_{z}(z)=B z+o(|z|) & \text { for } z \rightarrow 0,
\end{array}
$$

where $A, B: \mathbf{R}^{2 n} \rightarrow \mathbf{R}^{2 n}$ are two linear symmetric operators. Our aim is to give a lower bound for the number of nontrivial $\tau$-periodic solutions of (5-2) by appropriately comparing the operators $A$ and $B$ and the period $\tau$.

More precisely we are going to define an even integer number $\vartheta(\tau B / 2 \pi, \tau A / 2 \pi)$ which will give this lower bound. Given two hermitian operators $A, B: \mathbf{C}^{2 n} \rightarrow \mathbf{C}^{2 n}$ we set

$$
\begin{aligned}
N(A) & =\{\text { number of negative eigenvalues of } A\}, \\
\bar{N}(A) & =\{\text { number of nonpositive eigenvalues of } A\}, \\
\vartheta_{j}(B, A) & =N(i j J+B)-\bar{N}(i j J+A), \\
\vartheta(B, A) & =\sum_{j=-\infty}^{+\infty} \vartheta_{j}(B, A) .
\end{aligned}
$$

We observe that $\vartheta(B, A)$ is a finite number. In fact, for $j$ big enough $N(i j J+B)=$ $\bar{N}(i j J+A)=n$ and this implies that $\vartheta_{j}(B, A)=0$ except for a finite number of $j$ 's. Now we can state the main theorems of this section.

THEOREM 5.1. Suppose that $H$ satisfies $\left(\mathrm{H}_{1}\right),\left(\mathrm{H}_{2}\right)$ and

$\left(\mathrm{H}_{3}\right)(A z, z)>0 \forall z \in \mathbf{R}^{2 n} \backslash\{0\}$,

$\left(\mathrm{H}_{4}\right) H(z) \geqslant 0 \forall z \in \mathbf{R}^{2 n}$,

$\left(\mathrm{H}_{5}\right) \sigma(\tau J A / 2 \pi) \cap i \mathbf{Z}=\varnothing$.

Then equation (5-2) has at least $\frac{1}{2} \vartheta(\tau A / 2 \pi, \tau B / 2 \pi)$ nonconstant $\tau$-periodic solutions whenever $\vartheta(\tau A / 2 \pi, \tau B / 2 \pi) \geqslant 0$.

$\left(\mathrm{H}_{3}\right)$ is a technical assumption which will be discussed in Remark 5.9.

$\left(\mathrm{H}_{4}\right)$ is a condition which assures us that the periodic solutions we shall find are not constant (cf. the proof of Lemma 5.8).

If $\left(\mathrm{H}_{4}\right)$ is dropped, then we conclude that there are nonzero periodic solutions but they could be constant; therefore we lose information about their number (cf. Remark 4.4).

$\left(\mathrm{H}_{5}\right)$ is a "nonresonance condition at $\infty$ " and it expresses the fact that the linearized equation at $\infty$, i.e. $\dot{z}=J A z$ has no $\tau$-periodic solutions. If $\left(\mathrm{H}_{5}\right)$ does not hold, Theorem 5.1 is not any longer true unless we add some other conditions on $H$. For example we can add a condition which corresponds, roughly speaking, to the Landezman-Lazer condition for elliptic equations as is shown by the following theorem: 
THEOREM 5.2. Suppose that $H$ satisfies $\left(\mathrm{H}_{1}\right),\left(\mathrm{H}_{2}\right),\left(\mathrm{H}_{3}\right),\left(\mathrm{H}_{4}\right)$ and $\left(\mathrm{H}_{6}\right) V_{z}(z)$ is bounded where $V(z)=H(z)-\frac{1}{2}(A z, z)$.

$\left(\mathrm{H}_{7}\right) V(z) \rightarrow+\infty$ as $|z| \rightarrow+\infty$.

Then the conclusion of Theorem 5.1 holds.

Other assumptions can replace $\left(\mathrm{H}_{5}\right)$ as the theorem below shows.

THEOREM 5.3. Suppose that $H$ satisfies $\left(\mathrm{H}_{1}\right),\left(\mathrm{H}_{2}\right),\left(\mathrm{H}_{3}\right),\left(\mathrm{H}_{4}\right)$ and

$\left(\mathrm{H}_{8}\right)$ there is $R>0$ and $p \in(1,2)$ such that $p H(z) \geqslant\left(z, H_{z}(z)\right)>0$ for $|z| \geqslant R$.

$\left(\mathrm{H}_{9}\right) \varlimsup_{|z| \rightarrow+\infty}|z|^{-1}\left|H_{z}(z)\right| \leqslant \gamma<\frac{1}{2}$.

$\left(\mathrm{H}_{10}\right)$ there are constants $a_{1}>0$ and $a_{2}>0$ such that $H(z) \geqslant a_{1}|z|^{p}-a_{2}$.

Then the same conclusion of Theorem 5.1 holds.

REMARK 5.4. In order to give a feeling for the meaning of $\vartheta(\cdot, \cdot)$ we shall indicate some of its properties even if they will not be used in the proofs of the theorems. First of all, we observe that $\vartheta$ corresponds to the number $\frac{1}{2} \bar{k}$ defined by (4.1) as it will be shown in Lemma 6.6 .

Moreover it satisfies the following properties:

$\left(\vartheta_{1}\right)$ if $\vartheta(\lambda B, \lambda A) \geqslant 0$, then $\vartheta(\lambda A, \lambda B) \leqslant 0$.

$\left(\vartheta_{2}\right) \vartheta(\lambda A, \lambda A) \leqslant 0$.

$\left(\vartheta_{3}\right)$ if $B$ and $A$ satisfy the nonresonance condition i.e.

$$
\sigma(\lambda J A) \cap i \mathbf{Z}=\sigma(\lambda J B) \cap i \mathbf{Z}=\varnothing \quad\left(\text { cf. }\left(\mathrm{H}_{5}\right)\right)
$$

then

$$
\vartheta(\lambda B, \lambda A)=-\vartheta(\lambda A, \lambda B) .
$$

$\left(\vartheta_{4}\right)$ if $\sigma(J A) \cap i \mathbf{R}=\sigma(J B) \cap i \mathbf{R}=\varnothing$ then $\vartheta(\lambda B, \lambda A)=\vartheta(\lambda A, \lambda B)=0$.

$\left(\vartheta_{5}\right) \vartheta(\cdot, \cdot)$ is a symplectic invariant in both its arguments i.e.

$$
\vartheta\left(\lambda S_{1}^{T} A S_{1}, \lambda S_{2}^{T} B S_{2}\right)=\vartheta(\lambda A, \lambda B) \quad \forall S_{1}, S_{2} \in S_{\rho}(2 n) .
$$

Amann and Zehnder in [1] have introduced a symplectic invariant $\operatorname{Ind}(A, B, \alpha)$ with $\alpha$ real number. We have

$\left(\vartheta_{6}\right)$ if $\lambda B$ and $\lambda A$ satisfy $\left(\mathrm{H}_{5}\right)$ then

$$
\operatorname{Ind}\left(B, A, \lambda^{-1}\right)=\vartheta(\lambda B, \lambda A)
$$

and in general we have

$$
\left|\operatorname{Ind}\left(B, A, \lambda^{-1}\right)\right| \geqslant \vartheta(\lambda B, \lambda A) .
$$

Proof. $\left(\vartheta_{1}\right)$ and $\left(\vartheta_{2}\right)$ follow directly from the definition.

Let us prove $\left(\vartheta_{3}\right)$. Since $J^{2}=-$ Id we have that

$$
\operatorname{ker}(i j J+\lambda A)=\left\{v \in \mathbf{C}^{2 n} \mid \lambda J A v=j v\right\} .
$$

Therefore if $\left(\mathrm{H}_{5}\right)$ holds,

$$
\operatorname{ker}(i j J+\lambda A)=\{0\} \quad \text { and } N(i j J+\lambda A)=\bar{N}(i j J+\lambda A) .
$$

For the same reason $N(i j J+\lambda B)=\bar{N}(i j J+\lambda B)$.

Then $\left(\vartheta_{3}\right)$ follows from the definition. 
If $\sigma(J A) \cap i \mathbf{R}=\varnothing$, then $\operatorname{ker}(i j J+\lambda A)=\{0\} \forall \lambda \in \mathbf{R}$. Then, since the operator $i j J+\lambda A$ is not singular for any $\lambda \in \mathbf{R}$, the function

$$
\lambda \mapsto N(i J+\lambda A)=\bar{N}(i J+\lambda A)
$$

is constant. In particular it equals $n$ since for $\lambda=0$ we have that $N(i J)=n$. Arguing in the same manner we get that $N(i J+\lambda B)=\bar{N}(i J+\lambda B)=n$. Then $\left(\vartheta_{4}\right)$ follows.

Let us prove $\left(\vartheta_{5}\right)$. It is known that if $Q: \mathbf{C}^{2 n} \rightarrow \mathbf{C}^{2 n}$ is any hermitian operator and $S$ is any nonsingular operator

$$
N(Q)=N\left(S^{*} Q S\right) \text { and } \bar{N}(Q)=\bar{N}\left(S^{*} Q S\right)
$$

where $S^{*}$ is the adjoint of $S$.

If $S \in \mathrm{Sp}(2 n)$, then $S^{\mathrm{T}}=S^{*}$ (the transpose is equal to the adjoint since $S$ has real coefficients). Then using the above formula with $Q=i j J+\lambda A$ and $Q=i j J+\lambda B$, we get

$$
\bar{N}(i j J+\lambda A)=\bar{N}\left(i j S_{1}^{T} J S_{1}+\lambda S_{1}^{T} A S_{1}\right)=\bar{N}\left(i j J+\lambda S_{1}^{T} A S_{1}\right) \quad \forall S_{1} \in \operatorname{Sp}(2 n)
$$

and

$$
N(i j J+\lambda B)=N\left(i j J+\lambda S_{2}^{T} B S_{2}\right) \quad \forall S_{2} \in \operatorname{Sp}(2 n) .
$$

By the definition of $\vartheta(\cdot, \cdot)$ the conclusion follows.

$\left(\vartheta_{6}\right)$ follows from the characterization (5-16) of the eigenvalues of $i j J+\lambda A$ and easy computations.

REMARK 5.4'. If $A$ and $B$ commute with $J$ the number $\vartheta(\lambda B, \lambda A)$ is expressible in a form which is easy to compute. In fact let $\mu_{1} \cdots \mu_{2 n}$ be the set of eigenvalues of $A$ corresponding to the eigenvectors $v_{1}, \ldots, v_{2 n}$. Then the vectors $v_{k}+i J v_{k}$ are eigenvectors of the operator $i j J+\lambda A$ and they correspond to the eigenvalues $j+\lambda \mu_{k}$ $(k=1, \ldots, 2 n)$. Then setting $\mathscr{B}=\{1, \ldots, 2 n\}$ we get

$$
\bar{N}(i j J+\lambda A)=\#\left\{k \in \Re \mid j+\lambda \mu_{k} \leqslant 0\right\} .
$$

If the $\nu_{k}$ 's are the eigenvalues of $B$, arguing in the same manner we get

$$
N(i j J+\lambda B)=\#\left\{k \in \Re \mid j+\lambda \nu_{k}<0\right\} .
$$

Then, for $\lambda>0$, we have

$$
\begin{aligned}
\left\{k \in \mathscr{B} \mid \lambda \nu_{k}<-j\right\}= & \left\{k \in \mathscr{B} \mid \nu_{k}<\mu_{k} ; \lambda \mu_{k} \leqslant-j\right\} \\
& \cup\left\{k \in \mathscr{B} \mid \nu_{k}<\mu_{k} ; \lambda \nu_{k}<-j \leqslant \lambda \mu_{k}\right\} \\
& \cup\left\{k \in \mathscr{B} \mid \nu_{k} \geqslant \mu_{k} ; \lambda \nu_{k}<-j\right\}
\end{aligned}
$$

and

$$
\begin{aligned}
\left\{k \in \mathscr{B} \mid \lambda \mu_{k} \leqslant-j\right\}= & \left\{k \in \mathscr{B} \mid \nu_{k} \geqslant \mu_{k} ; \lambda \nu_{k}<-j\right\} \\
& \cup\left\{k \in \mathscr{B} \mid \nu_{k} \geqslant \mu_{k} ; \lambda \mu_{k} \leqslant-j \leqslant \lambda \nu_{k}\right\} \\
& \cup\left\{k \in \mathscr{B} \mid \nu_{k}<\mu_{k} ; \lambda \mu_{k} \leqslant-j\right\} .
\end{aligned}
$$

Thus

$$
\begin{aligned}
\vartheta_{j}(\lambda B, \lambda A)= & \#\left\{k \in \mathscr{B} \mid \nu_{k}<\mu_{k} \text { and } \lambda \nu_{k}<-j \leqslant \lambda \mu_{k}\right\} \\
& -\#\left\{k \in \mathscr{B} \mid \mu_{k} \leqslant \nu_{k} \text { and } \lambda \mu_{k} \leqslant-j \leqslant \lambda \nu_{k}\right\} .
\end{aligned}
$$


Therefore we have that

$$
\begin{aligned}
\vartheta(\lambda B, \lambda A)= & \#\left\{(j, k) \in \mathbf{Z} \times \mathscr{B} \mid \nu_{k}<\mu_{k} \text { and } \lambda \nu_{k}<j \leqslant \lambda \mu_{k}\right\} \\
& -\#\left\{(j, k) \in \mathbf{Z} \times \mathscr{B} \mid \nu_{k} \geqslant \mu_{k} \text { and } \lambda \mu_{k} \leqslant j \leqslant \lambda \nu_{k}\right\} .
\end{aligned}
$$

We remark that the number $\vartheta(\lambda A, \lambda B)$ does not depend on the order of the $\mu_{k}$ 's and the $\nu_{k}$ 's. If it is possible to order them in such a way that $\nu_{k}<\mu_{k}$ for each $k \in \mathscr{B}$, then

$$
\vartheta(\lambda B, \lambda A) \geqslant 0 \text { and } \lim _{\lambda \rightarrow+\infty}(\lambda B, \lambda A)=+\infty .
$$

This fact has an obvious interpretation in terms of the number of periodic solutions of $(5-2)$.

In order to prove Theorems 5.1, 5.2, 5.3 we shall use Theorem 4.6 (or Corollary 4.11). Before doing this some work is necessary.

First of all, making the change of variable

$$
t \mapsto \frac{2 \pi}{\tau} t=\lambda^{-1} t, \quad(5-2) \text { transforms to } \dot{z}=\lambda J H_{z}(z)
$$

and we seek $2 \pi$-periodic solutions of (5-3) which, of course, correspond to the $\tau$-periodic solutions of (5-2). We shall treat (5-3) in the Hilbert space $H=$ $H^{1 / 2}\left(S^{1}, \mathbf{R}^{2 n}\right)$, i.e. the space of $2 n$-ples of $2 \pi$-periodic functions which possess square integrable "derivative of order $\frac{1}{2}$ ". Perhaps the simplest way to introduce this space is as follows. Let $\bigodot^{\infty}\left(S^{1}, \mathbf{R}^{2 n}\right)$ be the space of $2 \pi$-periodic $n$-ples of $\bigodot^{\infty}$-functions. If $z \in e^{\infty}\left(S^{1}, \mathbf{R}^{2 n}\right)$ it has the following Fourier expansion

$$
z(t)=\frac{\alpha_{0}}{\sqrt{2 \pi}}+\frac{1}{\sqrt{\pi}} \sum_{j=0}^{+\infty}\left(\alpha_{j} \cos j t+\beta_{j} \sin j t\right), \quad \alpha, \beta \in \mathbf{R}^{2 n},
$$

which in complex notation becomes

$$
z(t)=\frac{1}{\sqrt{2 \pi}} \sum_{j=-\infty}^{+\infty} a_{j} e^{i j t} \quad \text { with } a_{j}=\bar{a}_{j} \in \mathbf{C}^{2 n} .
$$

$H^{1 / 2}\left(S^{1}, \mathbf{R}^{2 n}\right)$ is the closure of $e^{\infty}\left(S^{1}, \mathbf{R}^{2 n}\right)$ with respect to the Hilbert space norm

$$
\begin{aligned}
\|z\| & =\left[\left|\alpha_{0}\right|^{2}+\sum_{j=0}^{+\infty}(1+|\dot{J}|)\left(\left|\alpha_{j}\right|^{2}+\left|\beta_{j}\right|^{2}\right)\right]^{1 / 2} \\
& =\left[\sum_{j=-\infty}^{+\infty}(1+|j|)\left|a_{j}\right|^{2}\right]^{1 / 2} .
\end{aligned}
$$

$H^{1 / 2}\left(S^{1}, \mathbf{R}^{2 n}\right)$ can also be obtained by interpolation from the Sobolev spaces $H^{1}\left(S^{1}, \mathbf{R}^{2 n}\right)$ and $L^{2}\left(S^{1}, \mathbf{R}^{2 n}\right)$. From now on $H^{1 / 2}\left(S^{1}, \mathbf{R}^{2 n}\right)$ will be denoted by $H$. On $H$ a "canonical" representation of the group $S^{1}$ acts. If $s \in S^{1}$ ( $s$ is thought of as a real number in $[0,2 \pi)$ ) we have the representation

$$
T_{s} z(t)=z(t+s)
$$

here the " + " is the operation of the group $S^{1}$; i.e. the sum modulo $2 \pi$. 
In terms of the Fourier expansion (5-5), the representation $T_{s}$ has the following form:

$$
T_{s} z(t)=\sum_{j=-\infty}^{+\infty}\left(a_{j} e^{i j s}\right) e^{i j t}
$$

i.e. the Fourier expansion is a decomposition of $T_{s}$ in its irreducible components (see e.g. Weyl [18]).

We shall denote by $I=\{\Sigma, \mathfrak{T}, i\}$ the index theory relative to the representation $T_{s}$ described in II Example of $\$ 2$. The $2 \pi$-periodic solutions of $(5-3)$ correspond to the critical points in $H$ of the functional of the action:

$$
\begin{aligned}
f(z) & =-\int_{0}^{2 \pi}\left\{(p, \dot{q})_{\mathbf{R}^{n}}-\lambda H(z)\right\} d t \\
& =\int_{0}^{2 \pi}\left\{\frac{1}{2}(J \dot{z}, z)_{\mathbf{R}^{2 n}}+\lambda H(z)\right\} d t .
\end{aligned}
$$

(We have put the minus sign in front of the functional in order to make the notation consistent with the abstract theorems of $\S 4$.) If we set

$$
V(z)=H(z)-\frac{1}{2}(A z, z) \text { and } \Phi(z)=\lambda \int_{0}^{2 \pi} V(z) d t
$$

then we can write

$$
f(z)=\frac{1}{2} \int_{0}^{2 \pi}(J \dot{z}+\lambda A z, z)_{\mathbf{R}^{2 n}} d t+\Phi(z) .
$$

Let $L, \varphi: H \rightarrow H$ be the operators defined by the following formulas:

$$
\begin{aligned}
\langle L z, v\rangle & =\int_{0}^{2 \pi}(J \dot{z}+\lambda A z, v)_{\mathbf{R}^{2 n}} d t \quad \forall v \in H, \\
\langle\varphi(z), v\rangle & =\lambda \int_{0}^{2 \pi}\left(V_{z}(z), v\right) d t \quad \forall v \in H,
\end{aligned}
$$

then we have

$$
f(u)=\frac{1}{2}\langle L u, u\rangle+\Phi(u) \text { and } f^{\prime}(u)=L u+\varphi(u) .
$$

The following lemma holds.

Lemma 5.5. If the Hamiltonian function $H$ satisfies $\left(\mathrm{H}_{1}\right)$ and $\left(\mathrm{H}_{2}\right)$ then $f$ (defined by $(5-7))$ satisfies $\left(\mathrm{f}_{1}\right),\left(\mathrm{f}_{3}\right),\left(\mathrm{f}_{5}\right)(4-5)(\mathrm{a}),(\mathrm{d})$ and $(4-5)(\mathrm{c})$ where $Q$ is defined by the formula

$$
\langle Q z, v\rangle=\lambda \int_{0}^{2 \pi}(B z-A z, v) d t .
$$

Proof. By the Sobolev inequalities and standard arguments, it follows that $\varphi=\Phi^{\prime}$ is compact (see e.g. [7]). Then $f$ satisfies $\left(\mathrm{f}_{1}\right)$ and since $L$ and $\varphi$ are equivariant with respect to $T_{s}, f$ satisfies $\left(\mathrm{f}_{3}\right)$.

In order to prove $\left(f_{5}\right)$, it is enough to observe that the essential spectrum of the operator defined by the bilinear form

$$
(z, v) \mapsto \int_{0}^{2 \pi}\left(J \frac{d}{d t} z, v\right) d t
$$


is just $\{+1,-1\}$. Since $L$ is a compact perturbation of such operator, by well-known theorems (see e.g. [17]), it follows that

$$
\sigma_{0}(L)=\{+1,-1\} .
$$

(4-5)(a) follows by the definition of $\Phi$.

Let us prove (4-5)(d). By $\left(\mathrm{H}_{1}\right)$, for every $\varepsilon>0, \exists M>0$ such that

$$
\left|H_{z}(z)-A z\right| \leqslant \varepsilon|z|+M \text {. }
$$

Then, by (5-8), using the Hölder inequality we get

$$
\begin{aligned}
\left|\int_{0}^{2 \pi}\left(V_{z}(z), v\right) d t\right| & =\left|\int_{0}^{2 \pi}\left(H_{z}(z)-A z, v\right) d t\right| \\
& \leqslant \varepsilon\left|\int_{0}^{2 \pi}\right| z|\cdot| v|d t|+2 \pi M\|v\| \\
& \leqslant \varepsilon\|z\| \cdot\|v\|+2 \pi M\|v\|
\end{aligned}
$$

thus

$$
\begin{aligned}
\varlimsup_{\|z\| \rightarrow+\infty} \frac{\|\varphi(z)\|}{\|z\|} & =\lim _{\|z\| \rightarrow+\infty} \sup _{\|v\|=1} \frac{|\langle\varphi(z), v\rangle|}{\|z\|} \\
& =\lim _{\|z\| \rightarrow+\infty} \sup _{\|v\|=1} \frac{\lambda}{\|z\|}\left|\int_{0}^{2 \pi}\left(V_{z}(z), v\right) d t\right| \\
& \leqslant \lim _{\|z\| \rightarrow+\infty}\left(\lambda \varepsilon+\frac{2 \pi \lambda M}{\|z\|}\right)=\lambda \varepsilon
\end{aligned}
$$

By the arbitrariness of $\varepsilon,(4-5)(d)$ follows.

It remains to prove $(4-5)(\mathrm{c})$. By $\left(\mathrm{H}_{1}\right)$ we have that $\forall \varepsilon>0, \exists \delta_{1} \in(0,1)$ such that

$$
\left|H_{z}(z)-B z\right| \leqslant \varepsilon|z| \text { if }|z| \leqslant \delta_{1} \text {. }
$$

On the other hand, by $\left(\mathrm{H}_{2}\right), \exists \delta_{2}>1$, such that

$$
|H(z)-B z| \leqslant(|A|+|B|+1)|z| \text { for }|z|>\delta_{2}
$$

and by the continuity of $H_{z}(z)$ there is a constant $a_{1}>0$ such that

$$
\left|H_{z}(z)-B z\right| \leqslant a_{1} \text { for } \delta_{1} \leqslant|z| \leqslant \delta_{2} \text {. }
$$

By the above inequality we get

$$
\left|H_{z}(z)-B z\right| \leqslant \varepsilon|z|+a_{2}|z|^{2} \quad \text { with } a_{2}=\max \left(a_{1} \delta_{1}^{-2},|A|+|B|+1\right)
$$

$$
\text { for all } z \in \mathbf{R}^{2 n} \text {. }
$$

Then we have

$$
\begin{aligned}
\left|\int_{0}^{2 \pi}\left(H_{z}(z)-B z, v\right) d t\right| & \leqslant \int_{0}^{2 \pi}\left|H_{z}(z)-B z\right||v| d t \\
& \leqslant \varepsilon \int_{0}^{2 \pi}|z| \cdot|v| d t+a_{2} \int_{0}^{2 \pi}|z|^{2}|v| d t \\
& \leqslant \varepsilon\|z\| \cdot\|v\|+a_{2}\left(\int_{0}^{2 \pi}|z|^{4} d t\right)^{1 / 2} \cdot\|v\| \\
& \leqslant \varepsilon\|z\| \cdot\|v\|+a_{2}\|z\|_{L^{4}}^{2} \cdot\|v\|
\end{aligned}
$$


where $\|\cdot\|_{L^{4}}$ is the norm in $L^{4}\left(S^{1}, \mathbf{R}^{2 n}\right)$. By the Sobolev inequalities $\|z\|_{L^{4}} \leqslant a_{3}\|z\|$. Then we get

$$
\left|\int_{0}^{2 \pi}\left(H_{z}(z)-B z, v\right) d t\right| \leqslant \varepsilon\|z\| \cdot\|v\|+a_{2} a_{3}^{2}\|z\|^{2} \cdot\|v\| .
$$

Then using the above formula we have

$$
\begin{aligned}
|\langle\varphi(u)-Q u, v\rangle| & =\lambda\left|\int_{0}^{2 \pi}\left(V_{z}(z)-(B-A) z, v\right) d t\right| \\
& \leqslant \lambda\left|\int_{0}^{2 \pi}\left(H_{z}(z)-B z, v\right) d t\right| \quad \text { by }(6-8) \\
& \leqslant \lambda \varepsilon\|z\| \cdot\|v\|+\lambda a_{2} a_{3}^{2}\|z\|^{2} \cdot\|v\| .
\end{aligned}
$$

Then

$$
\varlimsup_{\|z\| \rightarrow 0} \frac{\|\varphi(z)-Q z\|}{\|z\|}=\varlimsup_{\|z\| \rightarrow 0} \sup _{\|v\|=1} \frac{\langle\varphi(z)-Q z, v\rangle}{\|z\|} \leqslant \lambda \varepsilon .
$$

By the arbitrariness of $\varepsilon,(4-5)(\mathrm{c})$ follows.

In order to apply Theorem 4.6 or Corollary 4.11 , we need the following lemma.

LEMMA 5.6. If $H$ satisfies $\left(\mathrm{H}_{1}\right)$ and $\left(\mathrm{H}_{2}\right)$, using the notation (5-8), (5-9), (5-9'), (4-4), and $\left(4-4^{\prime}\right)$ we have

$$
\operatorname{dim}\left(V^{+} \cap W^{-}\right)-\operatorname{cod}\left(V^{+}+W^{-}\right)=\vartheta(\lambda B, \lambda A) .
$$

In order to prove Lemma 5.6, we shall study the structure of the spaces defined by (4-4) and (4-4').

We start this program by studying the spectrum of the differential operators

$$
e_{z}=\left(J \frac{d}{d t}+\lambda A\right) z
$$

and

$$
\tilde{e} z=\left(J \frac{d}{d t}+\lambda B\right) z
$$

regarded as (unbounded) selfadjoint operators in $L^{2}\left(S^{1}, \mathbf{R}^{2 n}\right)$ since we have

$$
\langle L z, v\rangle=\int_{0}^{2 \pi}(\varrho z, v) d t \quad \forall z \in D(\varrho), \forall v \in H,
$$

$$
\text { (b) }\langle(L+Q) z, v\rangle=\int_{0}^{2 \pi}(\tilde{\mathscr{L}} z, v) d t \quad \forall z \in D(\tilde{\mathscr{L}}), \forall v \in H \text {. }
$$

Let us consider the following eigenvalue problem

$$
\left\{\begin{array}{l}
u \in \mathbf{C}^{2 n}, \\
(-i j J+\lambda A) u=\mu u, \quad i=\sqrt{-1}, j \in \mathbf{Z} .
\end{array}\right.
$$

The operator $i j J$ is a Hermitian operator, so there exists an orthonormal basis $\left\{u_{j k}\right\}$ ( $k=1, \ldots, 2 n ; j \in \mathbf{Z}$ ) of eigenvectors corresponding to real eigenvalues $\left\{\mu_{j k}\right\}$. If $u_{j k}$ is an eigenvector, then $\bar{u}_{j k}$ is also an eigenvector corresponding to the same eigenvalue. 
We claim that $u_{j k}$ cannot have all real components for $j \neq 0$. In fact we have

$$
i j\left(J u_{j k}, u_{j k}\right)+\left(A u_{j k}, u_{j k}\right)=\mu_{k}\left(u_{j k}, u_{j k}\right) \text {. }
$$

In the above equality, the second term and the right-hand side term are real; therefore $i j\left(J u_{j k}, u_{j k}\right)$ is real. Therefore, for $j \neq 0,\left(J u_{j k}, u_{j k}\right)$ must be a purely imaginary number, and this contradicts the fact that all the components of $u_{j k}$ are real. Then it follows that $u_{j k}$ and $\bar{u}_{j k}$ are linearly independent. Therefore every eigenvalue has even multiplicity (for $j \neq 0$ ). Also we can order the $u_{j k}$ 's in such a way that $u_{j k}$ corresponds to the same eigenvalue $u_{j k}$ as $u_{j, k+n}$ for $k=1, \ldots, n$.

Also we can suppose that

$$
\bar{u}_{j, k}=-i u_{j, k+n} \text { for } j \neq 0 .
$$

For $k=1, \ldots, 2 n$ and $j \in \mathbf{Z}$ we set

$$
u_{j k}=v_{j k}+i w_{j k} .
$$

Then by (5-12),

$$
\left\{\begin{array}{l}
\text { (a) } \quad v_{j, n+k}=w_{j k} \\
\text { (b) } \quad w_{j, n+k}=v_{j k}
\end{array} \text { for } j \neq 0 .\right.
$$

For $j=0$, we choose all the $u_{0, k}$ real, then we have

$$
u_{0, k}=v_{0, k}, \quad w_{0, k}=0 .
$$

Using elementary linear algebra we have

LEMMA 5.7. The $v_{j, k}$ 's are an orthonormal basis for $\mathbf{R}^{2 n}$ for each $j \in \mathbf{Z}$. Similarly the $w_{j, k}$ 's are an orthonormal basis for $\mathbf{R}^{2 n}$ for each $j \in \mathbf{Z} \backslash\{0\}$.

PROof. For $j \neq 0$ take a vector $\alpha \in \mathbf{R}^{2 n}$. In particular $\alpha \in \mathbf{C}^{2 n}$, so there are numbers $\alpha_{k}=\beta_{k}+i \gamma_{k} \in \mathbf{C}\left(\beta_{k}, \gamma_{k} \in \mathbf{R}\right)$ such that

$$
\alpha=\sum_{k=1}^{2 n} \alpha_{k} u_{j k}=\sum_{k=1}^{2 n}\left(\beta_{k} v_{j k}-\gamma_{k} w_{j k}\right)+i \sum_{k=1}^{2 n} \gamma_{k} v_{j k}+\beta_{k} w_{j k} .
$$

Since we have assumed $\alpha$ real, the last term vanishes and using (5-14) we have

$$
\alpha=\sum_{k=1}^{n} \beta_{k} v_{j k}-\gamma_{k} v_{j, n+k}+\sum_{k=1}^{n} \beta_{k+n} v_{j, n+k}-\gamma_{n+k} v_{j k} .
$$

This proves that the $v_{j, k}$ 's are a basis in $\mathbf{R}^{2 n}$.

The same argument shows that also the $w_{j k}$ form a basis in $\mathbf{R}^{2 n}$ for $j \neq 0$. For $j=0$ the conclusion follows by (5-15).

Using (5-11) and (5-13) we can write a formula which involves only real components:

$$
\left\{\begin{aligned}
j J w_{j k}+\lambda A v_{j k} & =\mu_{j k} v_{j k} \\
-j J v_{j k}+\lambda A w_{j k} & =\mu_{j k} w_{j k}
\end{aligned}\right.
$$

The above formula could give an alternative definition of the $v_{j, k}$ 's and the $w_{j, k}$ 's. We now set

$$
e_{j, k}=\frac{1}{\sqrt{2 \pi}}\left(v_{j k} \cos j t+w_{j k} \sin j t\right), \quad k=1, \ldots, 2 n \text { and } j \in \mathbf{Z} .
$$


It is easy to check that the $e_{j, k}$ 's are eigenfunctions of $\mathcal{L}$, i.e.

$$
\mathcal{L} e_{j, k}=\mu_{j k} e_{j k} \quad \text { for } k=1, \ldots, 2 n \text { and } j \in \mathbf{Z} \text {. }
$$

It is easy to see that the eigenspace

$$
H_{j, k}=\operatorname{Sp}\left\{e_{j, k}, e_{j, k+n}\right\}, \quad k=1, \ldots, n, j \in \mathbf{Z} \backslash\{0\},
$$

is $T_{s}$-invariant.

For $j=0$, we get that

$$
\operatorname{Sp}\left\{e_{0,1}, \ldots, e_{0,2 n}\right\}=F .
$$

Since the $u_{j, k}$ 's have been chosen normalized in $\mathbf{C}^{2 n}$, all the $e_{j, k}$ 's are normalized in $L^{2}\left(S^{1}, \mathbf{R}^{2 n}\right)$. Using the Fourier expansion (5-5) and the fact that the $v_{k}$ 's and the $w_{k}$ 's form a basis in $\mathbf{R}^{2 n}$ (Lemma 5.7), it is not difficult to show that the $e_{j, k}$ 's form an orthonormal basis in $L^{2}\left(S^{1}, \mathbf{R}^{2 n}\right)$.

Using (5-10) and (5-6') it is easy to see that the functions

$$
(1+|j|)^{-1} e_{j, k}, \quad j \in \mathbf{Z}, k=1, \ldots, 2 n,
$$

are the normalized eigenvectors of $L$ corresponding to the eigenvalues $(1+|j|)^{-1} \mu_{j k}$ and that they form an orthonormal basis in $H=H^{1 / 2}\left(S^{1}, \mathbf{R}^{2 n}\right)$.

Next we consider the eigenvalue problem

$$
\left\{\begin{array}{l}
u \in \mathbf{C}^{2 n}, \\
(i j J+\lambda B) u=\tilde{\mu} u .
\end{array}\right.
$$

Using the same arguments used for the eigenvalue problem (5-11) we can find a basis of normalized eigenfunctions of $\tilde{e}$

$$
\tilde{e}_{j, k}=\tilde{v}_{j k} \cos j t+\tilde{w}_{j k} \sin j t
$$

where $\tilde{v}_{j k}+i \tilde{w}_{j k}=\tilde{u}_{j k}$ are the eigenvectors of the problem (5-18). These eigenfunctions correspond to the eigenvalues $\tilde{\mu}_{j k}$ of the problem (5-18).

Then the functions $(1+|j|)^{-1} \tilde{e}_{j, k}$ are normalized in $H$. They are the eigenvectors of $L+Q$ and they correspond to the eigenvalues $(1+|j|)^{-1} \mu_{j k}$.

Finally we are able to characterize the spaces of the formulas (4-4) and (4-4').

In fact we have

$$
\begin{aligned}
& V^{ \pm}=\overline{\operatorname{Sp}\left\{e_{j, k}, e_{j, n+k} \mid j \in \mathbf{Z}, k=1, \ldots, n, \text { and } \mu_{j k} \gtrless 0\right\}}, \\
& V^{0}=\operatorname{Sp}\left\{e_{j, k}, e_{j, n+k} \mid j \in \mathbf{Z}, k=1, \ldots, n, \text { and } \mu_{j k}=0\right\}, \\
& W^{ \pm}=\overline{\operatorname{Sp}\left\{\tilde{e}_{j, k}, \tilde{e}_{j, n+k} \mid j \in \mathbf{Z}, k=1, \ldots, n, \text { and } \tilde{\mu}_{j k} \gtrless 0\right\},} \\
& W^{0}=\operatorname{Sp}\left\{\tilde{e}_{j, k}, \tilde{e}_{j k} \mid j \in \mathbf{Z}, k=1, \ldots, n, \text { and } \tilde{\mu}_{j k} \lessgtr 0\right\}
\end{aligned}
$$

(the closures are taken in $H$ ).

Now we are able to prove Lemma 5.6.

Proof of Lemma 5.6. We set

$$
\begin{aligned}
H_{j} & =\operatorname{Sp}\left\{e_{j k}, e_{j, k+n} \mid k=1, \ldots, n\right\}, \quad j \in \mathbf{Z}, \\
V_{j}^{ \pm} & =H_{j} \cap V^{ \pm} \quad \text { and } \quad W_{j}^{ \pm}=H_{j} \cap W^{ \pm} .
\end{aligned}
$$


By our construction we get

$$
\begin{aligned}
& \text { (a) } \operatorname{dim} W_{j}^{-}=N(i j J+\lambda B), \\
& \text { (b) } \operatorname{dim} V_{j}^{+}=2 n-\bar{N}(i j J+\lambda A) .
\end{aligned}
$$

By elementary linear algebra, we have

$$
\operatorname{dim}\left(V_{j}^{+}+W_{j}^{-}\right)=\operatorname{dim} V_{j}^{+}+\operatorname{dim} W_{j}^{-}-\operatorname{dim}\left(V_{j}^{+} \cap W_{j}^{-}\right)
$$

and, since $V_{j}^{+}$and $W_{j}^{-} \subset H_{j}$

$$
\begin{aligned}
\operatorname{dim}\left(V_{j}^{+}+W_{j}^{-}\right) & =\operatorname{dim} H_{j}-\operatorname{cod}_{H_{i}}\left(V_{j}^{+}+W_{j}^{-}\right) \\
& =2 n-\operatorname{cod}_{H_{j}}\left(V_{j}^{+}+W_{j}^{-}\right) .
\end{aligned}
$$

Combining (5-20) and (5-21) and using (5-19) we get:

$$
\begin{aligned}
\operatorname{dim}\left(V_{j}^{+} \cap W_{j}^{-}\right)- & \operatorname{cod}_{H_{j}}\left(V_{j}^{+}+W_{j}^{-}\right)=\operatorname{dim} V_{j}^{+}+\operatorname{dim} W_{j}^{-}-2 n \\
& =N(i j J+\lambda B)-\bar{N}(i j J+\lambda A)=\vartheta_{j}(\lambda B ; \lambda A) .
\end{aligned}
$$

Since $H=\bigoplus_{j \in \mathbf{z}} H_{j}$ we get

$$
\operatorname{dim}\left(V^{+} \cap W^{-}\right)-\operatorname{cod}_{H}\left(V^{+}+W^{-}\right)=\sum_{j} \vartheta_{j}(\lambda B ; \lambda A)=\vartheta(\lambda B, \lambda A) .
$$

Thus Lemma 5.6 is proved.

Lemma 5.8. If the Hamiltonian function satisfies $\left.\left(\mathrm{H}_{1}\right),\left(\mathrm{H}_{2}\right),\left(\mathrm{H}_{3}\right), \mathrm{H}_{4}\right)$ and the functional (5-7) satisfies (4-5)(b), then the equation (5-3) has at least $\frac{1}{2} \vartheta(\lambda B, \lambda A)$ nontrivial $2 \pi$-periodic solutions.

Proof. By our assumptions and Lemma 5.6, $f$ satisfies all the hypotheses of Theorem 4.6 except (4-5)(e). $F$ in our case is the space of the constants. By $\left(\mathrm{H}_{3}\right)$, $\mu_{0, k}>0$, so $F \subset V^{+}$. Now we can apply Theorem 4.6 and we can define $\bar{k}$ critical values with $\bar{k}=\frac{1}{2} \vartheta(\lambda B, \lambda A)$ via Lemma 5.6. It remains to prove that they do not correspond to constant functions (cf. Remark 4.4). We argue indirectly and we suppose that $z_{k} \in F$ is a critical point of $f$. Then, since all the critical values defined by Theorem 4.6 are negative we have

$$
f\left(z_{k}\right)=c_{k}<0 .
$$

By the definition of $f(6-7)$, we get

$$
f\left(z_{k}\right)=2 \pi \lambda H\left(z_{k}\right)
$$

and

$$
H\left(z_{k}\right)=(2 \pi \lambda)^{-1} c_{k}<0
$$

which contradicts $\left(\mathrm{H}_{4}\right)$. Thus the lemma is proved.

Proof of Theorem 5.1. We claim that if $\left(\mathrm{H}_{5}\right)$ holds, then $0 \notin \sigma(L)$.

We have proved that

$$
\sigma(L)=\left\{(1+|j|)^{-1} \mu_{j, k} \mid j \in \mathbf{Z} \text { and } k=1, \ldots, 2 n\right\} .
$$

Thus it is sufficient to show that

$$
0 \notin \sigma(i j J+\lambda A) \quad \text { with } \lambda=\frac{\tau}{2 \pi} .
$$


We argue indirectly and we suppose there is a $v \in \mathbf{C}^{2 n}$ such that

$$
i j J v+\lambda A v=0 \text {. }
$$

Then, applying $J$, we get

$$
\lambda J A v=i j v .
$$

Thus $i j \in \sigma(\lambda J A)=\sigma(\tau J A / 2 \pi)$ which contradicts $\left(\mathrm{H}_{5}\right)$. Then, from easy computations, (4-5)(b) follows (cf. Remark 4.10). Therefore we get the conclusion from Lemma 5.8.

Proof of TheOrem 5.2. Because of Lemma 5.8, it is enough to prove that the functional (5-7) satisfies (4-5)(b). We let $M_{1}, M_{2}, \ldots$ be positive constants. Let $z_{m}$ be a sequence such that

$$
f^{\prime}\left(z_{m}\right) \rightarrow 0
$$

We shall write $z_{m}=z_{m}^{+}+z_{m}^{0}+z_{m}^{-} \in V^{+} \oplus V^{0} \oplus V^{-}$.

By (5-22), for $m$ large enough, we get

$$
\left|\left\langle L z_{m}, z_{m}^{+}\right\rangle+\lambda \int_{0}^{2 \pi}\left(V_{z}(z), z_{m}^{+}\right) d t\right| \leqslant\left\|z_{m}^{+}\right\| .
$$

By $\left(\mathrm{H}_{6}\right),\left|V_{z}(z)\right| \leqslant M_{1}$. Then by the above inequality and (4-4)

$$
\beta\left\|z_{m}^{+}\right\|^{2} \leqslant\left|\left\langle L z_{m}, z_{m}^{+}\right\rangle\right| \leqslant 2 \pi \lambda M_{1}\left\|z_{m}^{+}\right\|+\left\|z_{m}^{+}\right\| \quad(\beta>0) .
$$

This gives a uniform bound for $z_{m}^{+}$. In the same manner, we can get a uniform bound for $z_{m}^{-}$. Moreover, since $f\left(z_{m}\right)$ is supposed to converge, it is bounded and

$$
\begin{aligned}
M_{2} & \geqslant f\left(z_{m}\right) \geqslant \lambda \int_{0}^{2 \pi} V\left(z_{m}\right) d t-\left|\left\langle L z_{m}, z_{m}\right\rangle\right| \\
& \geqslant \lambda \int_{0}^{2 \pi} V\left(z_{m}^{0}\right) d t+\lambda \int_{0}^{2 \pi}\left(V\left(z_{m}\right)-V\left(z_{m}^{0}\right)\right) d t-M_{3} \\
& \geqslant \lambda \int_{0}^{2 \pi} V\left(z_{m}^{0}\right) d t-\lambda M_{1} \int_{0}^{2 \pi}\left|z_{m}-z_{m}^{0}\right| d t-M_{3} \\
& \geqslant \lambda \int_{0}^{2 \pi} V\left(z_{m}^{0}\right) d t-2 \pi \lambda M_{1}\left\|z_{m}^{+}+z_{m}^{-}\right\|-M \geqslant \lambda \int_{0}^{2 \pi} V\left(z_{m}^{0}\right)-M_{4} .
\end{aligned}
$$

Therefore $\int_{0}^{2 \pi} V\left(z_{m}^{0}\right) d t$ is uniformly bounded by a constant $M_{5}$. Because of $\left(\mathrm{H}_{7}\right)$ there is a function $\chi: \mathbf{R}^{+} \rightarrow \mathbf{R}^{+}$such that

$$
\begin{aligned}
& \text { (a) } \chi(0)=0 ; \lim _{\varepsilon \rightarrow+\infty} \chi(t)=+\infty ; \chi^{\prime}(t)>0, \\
& \text { (b) } V(z) \geqslant \chi(|z|)-M_{6} .
\end{aligned}
$$

Since $V^{0}$ is a finite dimensional space, if $\left\|z_{m}^{0}\right\| \rightarrow+\infty$, then

$$
\int_{0}^{2 \pi} \chi\left(\left|z_{m}^{0}\right|\right) d t \rightarrow+\infty
$$

On the other hand, by $(5-23)(b)$, we have

$$
\int_{0}^{2 \pi} \chi\left(\left|z_{m}^{0}\right|\right) d t \leqslant \int_{0}^{2 \pi} V\left(z_{m}^{0}\right) d t+2 \pi \leqslant M_{5}+2 \pi_{6} .
$$

Therefore also the $z_{m}^{0}$ are uniformly bounded and the theorem is proved. 
Proof of Theorem 5.3. Because of Lemma 5.8, it is enough to show that the functional (5-7) satisfies (4-5)(b). This fact is shown in [7] in the proof of Corollary 4.11.

REMARK 5.9. Amann and Zehnder [2] have estimated the number of $\tau$-periodic solutions for uniformly convex Hamiltonians which satisfy $\mathrm{H}_{1}, \mathrm{H}_{2}$ and other technical assumptions. Under $\left(\mathrm{H}_{5}\right)\left(\mathrm{cf} .\left(\vartheta_{3}\right)\right)$, this number is greater or equal to

$$
\max \left\{\operatorname{Ind}\left(B, A, \lambda^{-1}\right) ; \operatorname{Ind}\left(A, B, \lambda^{-1}\right)\right\} \quad\left(\lambda=\frac{\tau}{2 \pi}\right)
$$

which, by $\left(\vartheta_{6}\right)$ and $\left(\vartheta_{3}\right)$, equals $|\vartheta(\lambda B, \lambda A)|$.

Their proof reduces the problem to a finite dimensional one with no fixed points. Our proof works directly in infinite dimensional setting. Moreover we have treated more general Hamiltonians, at the expense of the condition

$$
\vartheta(\lambda B, \lambda A)>0
$$

which we needed to control $F$ and get meaningful results in Theorems 5.1, 5.2 and 5.3.

Adding the further symmetry property $H(z)=H(-z)$ to the Hamiltonian the space of fixed points $F$ reduces to $\{0\}$ and the previous theorem can be improved as is shown in Theorem 5.10.

In addition to the result of Amann and Zehnder and Theorem 5.10, other results are available when $\vartheta(\lambda B, \lambda A)<0$. If the Hamiltonian function has the form

$$
H(p, q)=\frac{1}{2} p^{2}+V(q)
$$

an estimate of the number of $\tau$-periodic solution given in [6] using the fact that the corresponding variational problem was semidefinite. For example, under $\left(\mathrm{H}_{1}\right),\left(\mathrm{H}_{2}\right)$, $\left(\mathrm{H}_{4}\right)$ and $V(q) \leqslant 0$ for every $q \in \mathbf{R}^{n}$ such that $\partial V(q) / \partial q=0$ it was shown (Theorem 4.4) that (5.2) possesses at least $\frac{1}{2} \vartheta(\lambda A, \lambda B)$ nonconstant periodic solutions (actually the number of solutions was estimated by comparing the eigenvalues of $\partial^{2} V / \partial q^{2}$ at 0 and at $\infty$; but it is not difficult to show that this number equals $\left.\frac{1}{2} \vartheta(\lambda A, \lambda B)\right)$.

We conclude this section with

TheOREM 5.10. Suppose that $H$ satisfies $\left(\mathrm{H}_{1}\right),\left(\mathrm{H}_{2}\right),\left(\mathrm{H}_{5}\right)$ and

$$
H(z)=H(-z) \text {. }
$$

Then the equation (5-2) has at least $\bar{k}$ nonzero independent $\tau$-periodic solutions where

$$
\bar{k}=\frac{1}{2} \max \left\{\vartheta\left(\frac{\tau}{2 \pi} B, \frac{\tau}{2 \pi} A\right) ; \vartheta\left(\frac{\tau}{2 \pi} A, \frac{\tau}{2 \pi} B\right)\right\} .
$$

Proof. We consider the space

$$
H^{1 / 2}\left(S^{1}, \mathbf{R}^{2 n}\right)
$$

with the action of the antipodal mapping described in the examples of $\S 2$.

If $\vartheta(\lambda B, \lambda A) \geqslant \vartheta(\lambda A, \lambda B)(\lambda=\tau / 2 \pi)$ then we argue in the same manner we have done for proving Lemma 5.8 and Theorem 5.1. We do not need $\left(\mathrm{H}_{3}\right)$ since it was used only to prove that $F \subset V^{+}$while here $F=\{0\}$. Also we do not need $\left(\mathrm{H}_{4}\right)$ since it has been used only for proving that the critical values do not correspond to constant functions. 
Therefore we get the same conclusion as Theorem 4.6 and since in this case $d=1$, we get $\vartheta(\lambda B, \lambda A)$ critical values. However, if they correspond to nonconstant periodic solutions, they are degenerate, i.e. $i\left(\mathbf{K}_{c}\right)=2$ (or a multiple of 2). If we want to count the independent periodic solutions (i.e. if we identify the solutions obtained from each other by a time translation) we have to divide this number by 2 .

If $\vartheta(\lambda A, \lambda B)>\vartheta(\lambda B, \lambda A)$ we argue in the same way replacing the functional (6-7) with its negative.

REMARK 5.11. We have not used $\left(\mathrm{H}_{4}\right)$, which was used in the proof of Lemma 5.8 to show that we did not get constant solutions. In fact the conclusion of Theorem 5.10 is about nonzero periodic solutions, but it might happen that there are some constant solutions.

REMARK 5.12. Theorem 5.10 could have been applied also to the nonautonomous case for a $\tau$-periodic Hamiltonian function since we have exploited only the evenness and not the time translation invariancy. Then, instead of getting $\bar{k}$ independent families of $\tau$-periodic solutions we would just get $2 \bar{k}$ pairs of periodic solutions.

6. Remarks on superquadratic Hamiltonian systems. The Hamiltonian system (5-2) is called superquadratic if

$$
\left\{\begin{array}{l}
\text { there is a constant } p \in(2,+\infty) \text { and } R>0 \text { such that } 0< \\
p H(z) \leqslant\left(H_{z}(z), z\right) \text { when }|z|>R .
\end{array}\right.
$$

In [16] the existence of at least one periodic solution for any $\tau>0$ was established for superquadratic Hamiltonian systems.

This fact of course implies that (5-2) possesses infinitely many $\tau$-periodic solutions since a $\tau / l$-periodic solution is also a $\tau$-periodic solution for any $l \in \mathbf{N}^{+}$. Thus if we apply our abstract theorems, we do not improve such known results for autonomous superquadratic Hamiltonian systems.

However, when $H$ is even and time dependent Theorem 4.6 can be applied to get a new result:

Theorem 6.1. Suppose $H(t, z)=\frac{1}{2}(A z, z)+\hat{H}(t, z)$ with $A: \mathbf{R}^{2 n} \rightarrow \mathbf{R}^{2 n}$ is a symmetric operator and $\hat{H}$ satisfies

$$
\begin{cases}\text { (a) } & \hat{H}(t, z) \geqslant 0 \forall t \in \mathbf{R}, \forall z \in \mathbf{R}^{2 n} . \\ \text { (b) } & \hat{H}(t, z)=o\left(|z|^{2}\right) \text { as }|z| \rightarrow 0 . \\ \text { (c) } & \text { there is } p \in(2,+\infty) \text { and } R>0 \text { such that } \\ & 0<p \hat{H}(t, z) \leqslant\left(\hat{H}_{z}(z), z\right), \forall t \in \mathbf{R} \text { and }|z| \geqslant R . \\ \text { (d) } & \text { there is } \tau>0 \text { such that } \hat{H}(t+\tau, z) \\ & =\hat{H}(t, z) \forall t \in \mathbf{R}, \forall z \in \mathbf{R}^{2 n} \text {. } \\ \text { (e) } & \text { there are constants } \alpha, R_{1}>0 \text { such that } \\ & \left|\hat{H}_{z}(t, z)\right| \leqslant \alpha\left(z, H_{z}(t, z)\right), \forall t \in \mathbf{R},|z|>R_{1} . \\ \text { (f) } & \hat{H}(t, z)=\hat{H}(t,-z) .\end{cases}
$$

Moreover suppose that the eigenvalue problem

$$
A \xi=\lambda J \xi
$$


has $2 n$ purely imaginary eigenvalues different from 0 . Then the Hamiltonian system

$$
\dot{z}=J H_{z}(t, z)
$$

has infinitely many $\tau$-periodic solutions.

In [14] Rabinowitz proved that the system (6-4) has at least one $\tau$-periodic solution without requiring (6-2)(f). Adding the symmetry property (6-2)(f) to $\hat{H}$, we can prove that (6-4) has infinitely many periodic solutions. The proof is a straightforward application of Corollary 6.5, Lemma 4.8 and the estimates of [14]; so we shall not carry out the details.

\section{REFERENCES}

1. H. Amann and E. Zehnder, Nontrivial solutions for a class of nonresonance problems and applications to nonlinear differential equations, Ann. Scuola Norm. Sup. Pisa Cl. Sci. (4) 9 (1980), 539-603.

2. Multiple periodic solutions of asymptotically linear Hamiltonian equations, Manuscripta Math. 32 (1980), 149-189.

3. A. Ambrosetti and P. H. Rabinowitz, Dual variational methods in critical point theory and applications, J. Funct. Anal. 14 (1973), 349-381.

4. P. Bartolo, V. Benci and D. Fortunato, Abstract critical point theorems and applications to some nonlinear problems with strong resonance at infinity (to appear).

5. V. Benci, Some critical point theorems and applications, Comm. Pure Appl. Math. 33 (1980).

6. __ A geometrical index for the group $S^{1}$ and some applications to the study of periodic solutions of ordinary differential equations, Comm. Pure Appl. Math. 34 (1981), 393-432.

7. V. Benci and P. M. Rabinowitz, Critical point theorems for indefinite functionals, Invent. Math. 52 (1979), 241-273.

8. D. C. Clark, A variant of Ljiusternik Schnirelmann theory, Indiana Univ. Math. J. 22 (1972), 65-74.

9. I. Eckeland and J. M. Lasry, Sur le nombre de points critiques de fonctions invariantes par des groupes, C. R. Acad. Sci. Paris Sér. A 282 (1976), 559-562.

10. E. Fadell and P. H. Rabinowitz, Generalized cohomological index theories for Lie group action with an application to bifurcation questions for Hamiltonian systems, Invent. Math. 45 (1978), 134-174.

11. M. A. Krasnoselskii, Topological methods in the theory of nonlinear integral equations, Macmillan, New York, 1964.

12. L. A. Ljusternik and L. Schnirelmann, Topological methods in the calculus of variations, Hermann, Paris, 1934.

13. P. H. Rabinowitz, A variational method for nonlinear eigenvalue problems, Nonlinear Eigenvalue Problems (G. Prodi, editor), Edizione Cremonese, Roma, 1974, pp. 141-195.

14. On subharmonic solutions of Hamiltonian systems, Comm. Pure. Appl. Math. 33 (1980), $609-633$.

15. Periodic solutions of Hamiltonian systems, Comm. Pure Appl. Math. 31 (1978), 225-251.

16. _ Variational methods for finding periodic solutions of differential equations, Nonlinear Evolution Equations (M. Crandall, editor), Academic Press, New York, 1978, pp. 225-251.

17. M. Schechter, Spectra of partial differential operators, North-Holland, Amsterdam, 1971.

18. H. Weyl, The theory of groups and quantum mechanics, Dover, New York, 1950.

Istituto di Matematica ApPlicata, Universita di Bari, Bari, Italy

Current address: Mathematics Research Center-University of Wisconsin, 610 Walnut Street, Madison, Wisconsin 53706 